\title{
Btk-specific inhibition blocks pathogenic plasma cell signatures and myeloid cell-associated damage in IFN $\alpha$-driven lupus nephritis
}

\author{
Arna Katewa, ${ }^{1}$ Yugang Wang, Jason A. Hackney, ${ }^{2}$ Tao Huang, ${ }^{1}$ Eric Suto, ${ }^{3}$ Nandhini Ramamoorthi, ${ }^{4}$ \\ Cary D. Austin, ${ }^{5}$ Meire Bremer, ${ }^{6}$ Jacob Zhi Chen, ${ }^{7}$ James J. Crawford, ${ }^{8}$ Kevin S. Currie, ${ }^{9}$ \\ Peter Blomgren, ${ }^{9}$ Jason DeVoss, ${ }^{3}$ Julie A. DiPaolo, ${ }^{10}$ Jonathan Hau, ${ }^{11}$ Adam Johnson, ${ }^{12}$ Justin Lesch, ${ }^{3}$ \\ Laura E. DeForge, ${ }^{12}$ Zhonghua Lin, ${ }^{3}$ Marya Liimatta,, ${ }^{12}$ Joseph W. Lubach, ${ }^{11}$ Sami McVay, ${ }^{12}$ \\ Zora Modrusan, ${ }^{13}$ Allen Nguyen, ${ }^{12}$ Chungkee Poon, ${ }^{1}$ Jianyong Wang, ${ }^{12}$ Lichuan Liu, ${ }^{14}$ Wyne P. Lee, ${ }^{3}$ \\ Harvey Wong, ${ }^{7}$ Wendy B. Young, ${ }^{8}$ Michael J. Townsend, ${ }^{4}$ and Karin Reif ${ }^{1}$ \\ 'Departments of Immunology Discovery, ${ }^{2}$ Bioinformatics and Computational Biology, ${ }^{3}$ Translational Immunology, \\ ${ }^{4}$ Biomarker Discovery OMNI, ${ }^{5}$ Pathology, ${ }^{6}$ Biomarker Development, ${ }^{7}$ Drug Metabolism and Pharmacokinetics, ${ }^{8}$ Discovery \\ Chemistry, at Genentech, South San Francisco, California, USA. ${ }^{9}$ Gilead Sciences, Seattle, Washington, USA. ${ }^{10} \mathrm{Gilead}$ \\ Sciences, Foster City, California, USA. "15mall Molecule Pharmaceutical Sciences, ${ }^{12 B i o c h e m i c a l ~ a n d ~ C e l l u l a r ~ P h a r m a c o l o g y, ~}$ \\ ${ }^{13}$ Molecular Biology, ${ }^{14}$ Clinical Pharmacology at Genentech, South San Francisco, California, USA
}

Systemic lupus erythematosus (SLE) is often associated with exaggerated B cell activation promoting plasma cell generation, immune-complex deposition in the kidney, renal infiltration of myeloid cells, and glomerular nephritis. Type-I IFNs amplify these autoimmune processes and promote severe disease. Bruton's tyrosine kinase (Btk) inhibitors are considered novel therapies for SLE. We describe the characterization of a highly selective reversible Btk inhibitor, G-744. G-744 is efficacious, and superior to blocking BAFF and Syk, in ameliorating severe lupus nephritis in both spontaneous and IFN $\alpha$-accelerated lupus in NZB/W_F1 mice in therapeutic regimens. Selective Btk inhibition ablated plasmablast generation, reduced autoantibodies, and - similar to cyclophosphamide - improved renal pathology in IFNo-accelerated lupus. Employing global transcriptional profiling of spleen and kidney coupled with cross-species human modular repertoire analyses, we identify similarities in the inflammatory process between mice and humans, and we demonstrate that G-744 reduced gene expression signatures essential for splenic B cell terminal differentiation, particularly the secretory pathway, as well as renal transcriptional profiles coupled with myeloid cell-mediated pathology and glomerular plus tubulointerstitial disease in human glomerulonephritis patients. These findings reveal the mechanism through which a selective Btk inhibitor blocks murine autoimmune kidney disease, highlighting pathway activity that may translate to human SLE.

Authorship note: AK, YW, and JAH contributed equally to this work. MJT and KR are co-senior authors.

Conflict of interest: All authors (except $\mathrm{KC}, \mathrm{PB}$, and $\mathrm{JD}$ ) are or were employed by Genentech Inc. at the time of the study and hold equity in the Roche Group. KC, PB, and JD were employed by CGI Pharmaceuticals. In addition, the research in this manuscript was fully funded by Cenentech Inc.

Submitted: August 12, 2016 Accepted: February 23, 2017 Published: April 6, 2017

Reference information: JCI Insight. 2017;2(7):e90111. https:// doi.org/10.1172/jci.insight.90111.

\section{Introduction}

Systemic lupus erythematosus (SLE) is a complex autoimmune disease characterized by breakdown of immune cell tolerance, activation of autoreactive $\mathrm{T}$ and $\mathrm{B}$ cells, production of antinuclear antibodies (ANA), and deposition of immune-complexes (IC) leading to recruitment of inflammatory cells (1). Alterations in both innate and adaptive arms of the immune system promote disease progression and organ damage.

B cells play a central role in lupus pathogenesis through the production of autoantibodies that recognize nuclear components, by generation of proinflammatory cytokines, including IL- 6 and IL-10, and through $\mathrm{T}$ cell activation (2). Myeloid cells and DCs also contribute to the breakdown in peripheral tolerance and, thus, disease progression (3). Lupus nephritis (LN) is a common and potentially devastating manifestation of lupus that occurs in more than half of SLE patients. Renal disease in lupus is associated with significant morbidity and mortality. LN is characterized by renal IC deposition and infiltration with mononuclear phagocytes that, in humans, correlate with poor disease outcome and are associated 
with glomerular cytokine/chemokine production, complement activation, and extensive proteinuria $(4,5)$. In NZB/W_F1 SLE-prone mice, direct activation of Fc $\gamma$ receptor-bearing (Fc $\gamma$ R-bearing) myeloid cells, including monocytes/macrophages, by glomerular ICs is sufficient to initiate inflammatory responses, resulting in tissue damage (6). The autoantibody IC also activate TLRs 7 and 9 in myeloid cells and plasmacytoid DCs, leading to the secretion of IFN $\alpha$ that amplifies immune responses and consequently worsens disease $(7,8)$. IFN $\alpha$ augments B cell abnormalities in conjunction with TLR stimulation by lowering the activation threshold of autoreactive B cells, enhancing their survival and differentiation into plasmablasts and thereby triggering an excessive germinal center (GC) response $(1,2,9,10)$. In human SLE patients, enhanced IFN $\alpha$ stimulation, demonstrated through an IFN gene signature in blood, correlates with disease severity and higher ANA levels (11). Studies in NZB/W_F1 mice have confirmed the enhancing function of type-I IFNs in lupus pathogenesis. NZB/W_F1 mice deficient in type-I IFN receptor show prolonged survival (12), and conversely, adenovirus-mediated delivery of IFN $\alpha$ accelerates lupus manifestations, leading to severe glomerulonephritis $(5,13,14)$.

Current treatments for severe SLE or LN, such as mycophenolate mofetil or cyclophosphamide (CTX), are effective at reducing mortality but fail to provide a cure, and they are accompanied by severe adverse effects via their immunosuppressive or cytotoxic properties, respectively $(15,16)$. The only targeted immunotherapy approved for SLE is the anti-BAFF Ab belimumab that acts by reducing naive and transitional B cells (17). However, initial clinical trials were not designed to assess the efficacy of belimumab for the treatment of LN. B cell depletion through anti-CD20 treatment has been studied in lupus, substantiating pathogenic roles of B cells, but clinical trials of anti-CD20 in SLE and LN have not supported approval (2, 9). Therefore, there is a high unmet need for targeted therapy in SLE. Because of the complexity of B cell involvement in disease pathogenesis, a drug that antagonizes more than one effector pathway would hold great therapeutic potential for more severe disease.

Bruton's tyrosine kinase (Btk) is a Tec-family kinase that is expressed in most hematopoietic cells but not $\mathrm{T}$ cells. Btk is a key mediator of $\mathrm{B}$ cell receptor $(\mathrm{BCR})$ signaling in $\mathrm{B}$ cells and $\mathrm{Fc} \gamma \mathrm{R}$ signaling in myeloid cells (18-20). Mutations in the Btk gene lead to B cell deficiency manifested as X-linked agammaglobulinemia in humans and the related but less severe X-linked immunodeficiency in mice, emphasizing its role in B cell development. In animal models of arthritis, Btk inhibition abrogates both B cell- and myeloid cell-mediated disease marked by reductions in autoantibody and inflammatory cytokine levels $(18,21,22)$. Furthermore, Btk deficiency and Btk inhibitors such as RN486, M7583, BI-BTK-1, and PF-06250112 of divergent selectivity profiles have shown benefit in preclinical models of SLE (23-30).

Given the fundamental role of Btk in both B cell and myeloid cell function and the chronic nature of treatment in autoimmune disorders, highly selective Btk inhibition is an attractive therapeutic strategy for SLE. Here, we show that specific Btk inhibition with a new prototype noncovalent, reversible Btk inhibitor G-744 - which exhibits over 400-fold selectivity against 284 kinases tested - abrogates aggressive disease in IFN $\alpha$-accelerated NZB/W_F1 SLE and is superior to BAFF blockade or Spleen tyrosine kinase (Syk) inhibition. Furthermore, transcriptional profiling and modular expression analyses of spleen and kidney revealed that Btk inhibition, but not BR3-Fc or a Syk inhibition, abrogates gene signatures associated with plasma cell (PC) generation in the spleen, myeloid cell activation in the kidney, and decreased the expression of renal ortholog genes previously identified to show enhanced expression in the glomerular and tubulointerstitial compartments in human LN patients.

\section{Results}

G-744 is a noncovalent, highly selective small molecule inhibitor for Btk. We previously reported CGI-1746 as a highly selective, noncovalent, reversible inhibitor of Btk (18). Further lead optimization efforts identified the noncovalent, reversible Btk inhibitor G-744 (Figure 1A) (31) that had a similar binding mode and selectivity profile as CGI-1746 but improved physiochemical, absorption, distribution, metabolism, and excretion properties, allowing for oral dosing. In in vitro enzyme assays, G-744 potently inhibited Btk kinase activity with an $50 \%$ inhibitory concentration $\left(\mathrm{IC}_{50}\right)$ of $1.28 \mathrm{nM}$ at apparent $\mathrm{K}_{\mathrm{m}}$ ATP concentration (Table 1). G-744 demonstrated > 1,000-fold biochemical selectivity over all 284 kinases tested except for EphA7 and Fgr, which still showed robust selectivity of 428-fold and 868-fold, respectively (Table 1 and Supplemental Table 1; supplemental material available online with this article; https://doi.org/10.1172/ jci.insight.90111DS1). We compared G-744 with other Btk inhibitors and showed that G-744 exhibited the highest selectivity against Tec-family kinases (Itk, Tec) and Src-family kinases (Blk, Frk, Hck, Lck, Lyn, 
A<smiles>Cn1cc(-c2cccc(N3CCc4c(sc5c4CC(C)(C)C5)C3=O)c2CO)cc(Nc2ccncn2)c1=O</smiles>

B

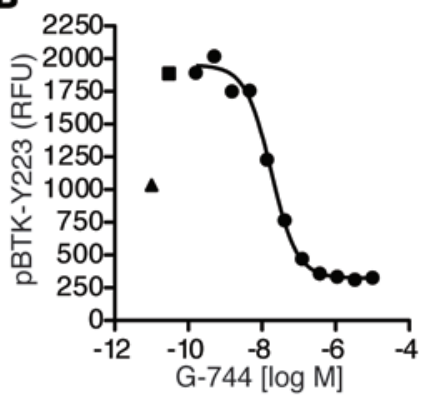

C

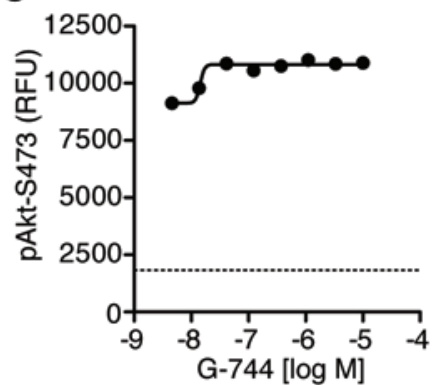

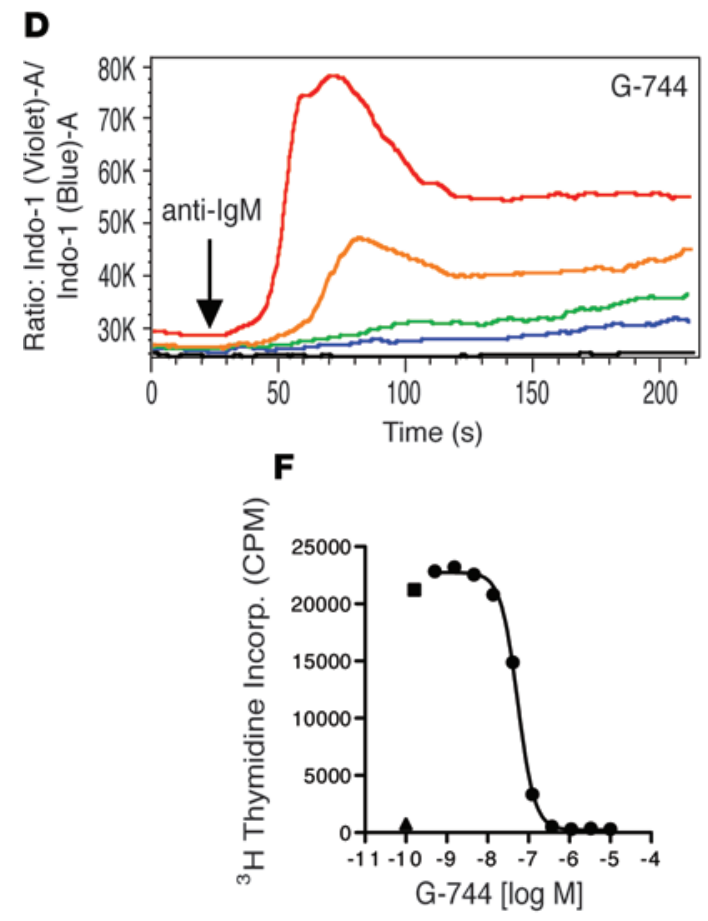

E

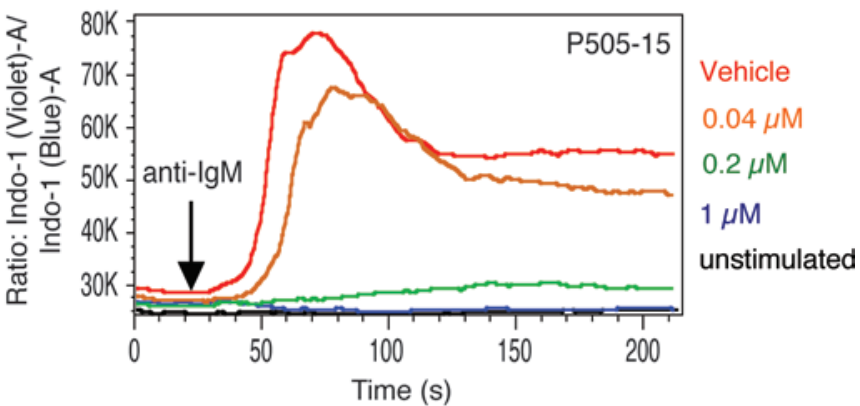

G

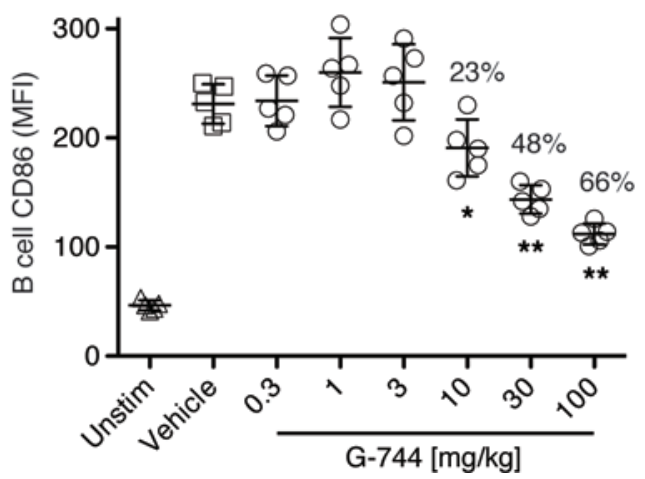

Figure 1. G-744 inhibits Btk signaling and B cell receptor-mediated (BCR-mediated) responses in murine B cells. (A) Chemical structure of G-744. (B-F) $B$ cell signaling readouts in presence of indicated concentrations of G-744 or P505-15 following anti-lgM $F\left(a^{\prime}\right)_{2}$, stimulation of primary murine splenic $B$ cells. (B) Phosphorylation of Btk-Y223 in presence of G-744. The triangle indicates pBtk-Y223 phosphorylation in unstimulated B cells without G-744. (C) Phosphorylation of Akt-S473 in presence of G-744. (D and E) Induction of [Ca2+] in presence of (D) G-744 or (E) P505-15. Arrows indicate stimuli added. (F) Proliferation in presence of G-744. The triangle and square indicate B cell proliferation without G-744 in un- and anti-lgM F(ab') $)_{2}$-stimulated B cells, respectively. (G) Inhibition of anti-IgD-induced CD86 induction in C57BL/6 mice by G-744. CD86 expression on splenic B220 ${ }^{+}$B cells was quantified by flow cytometry $6 \mathrm{~h}$ after anti-lgD stimulation. Mice were dosed orally with vehicle or G-744 as indicated prior to anti-lgD injection. Mean fluorescence intensity (MFI) is indicated for individual mice (represented by symbols), and data is expressed as mean \pm SEM for each group $(n=5)$. Percent inhibition by G-744 treatment compared with stimulated vehicle control is indicated. ${ }^{*} P<0.05$, ${ }^{* *} P<0.0001$, one-way ANOVA with Dunnett's correction. (B-G) Graphs are representative of at least 3 experiments.

Src, and Yes), as well as several other kinases including Csk, EGFR, Flt3, and Jak3 (Supplemental Table 1). These results demonstrated that G-744 is a Btk inhibitor with a favorable selectivity profile that allows for a direct examination of the contribution of Btk to cellular functions and autoimmune disease pathogenesis independent of coinhibition of other enzymes, including Tec- and Src-family kinases.

G-744 inhibits murine B cell and macrophage signaling. We evaluated the cellular potency and functional activity of G-744 and compared it with a selective Syk inhibitor P505-15 (32) (Supplemental Table 1), since both Btk and Syk play a critical role in signal transduction of BCR and FcR $(21,33)$. G-744 potently inhibited anti-IgM-induced Btk-Y223 tyrosine phosphorylation (pBtk) in murine B cells (Figure 1B and Table 2) with an $\mathrm{IC}_{50}$ of $20 \mathrm{nM}$. As expected, Btk inhibition had no effect on BCR-induced Akt phosphorylation on S473 (Figure 1C) (34). G-744 abrogated anti-IgM-induced $\mathrm{Ca}^{2+}$ flux (Figure 1D) and murine B cell proliferation (Figure $1 \mathrm{~F}$ and Table 2). Upregulation of the $\mathrm{T}$ cell costimulatory molecule CD86 on B cells after 

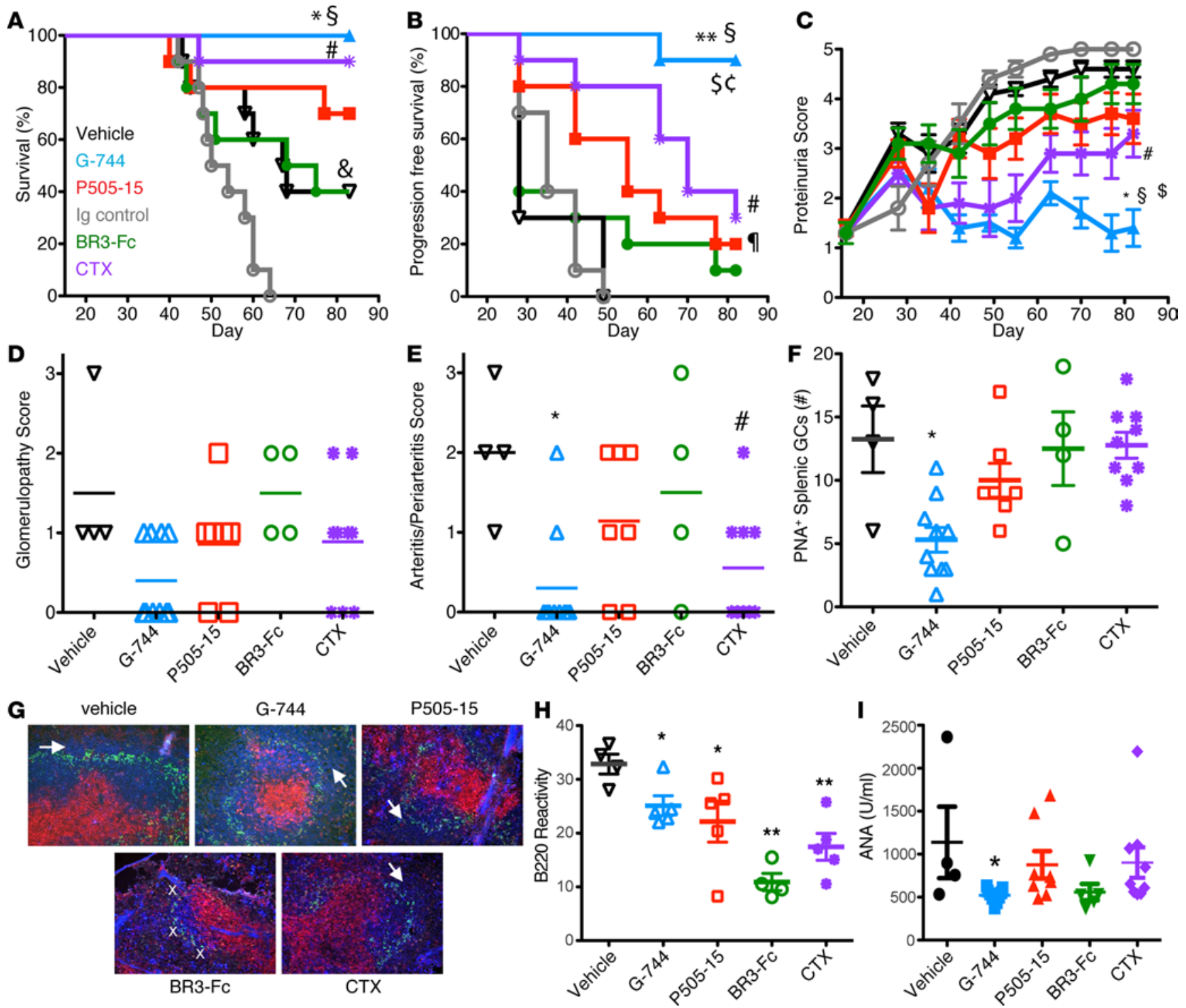

Figure 2. Specific Btk inhibition is superior to Syk or BAFF blockade in improving disease in IFNo-enhanced NZB/W_F1 LN. Fifteen-week-old NZB/W_F1 female mice ( $n=10$ /group) were administered 1 injection of adenovirus containing murine IFN $\alpha$ (AdIFN $\alpha$ ). Drug treatment started 3 weeks after AdIFN $\alpha$ injection with $100 \mathrm{mg} / \mathrm{kg}$ G-744 (triangles, dark-blue line), $60 \mathrm{mg} / \mathrm{kg}$ P505-15 (squares, red line), vehicle Hydroxypropylmethyl cellulose (HPMC) (open triangles, black line), $7.5 \mathrm{mg} / \mathrm{kg}$ BR3-Fc (circles, green line), Ig control antibodies (open circles, grey line), or Cyclophosphamide (CTX, stars, magenta line) as described in Methods. Graphs show (A) percentage of overall survival, (B) percentage progression-free survival, and (C) proteinuria score as measure of clinical efficacy. (D-H) Effect of drug treatment after 62 days at end of study in surviving mice (83 days after AdIFN $\alpha$ induction) on (D) renal glomerulopathy; (E) renal arteritis/periarteritis; (F) splenic peanut agglutinin (PNA $)$ germinal centers (GCs) (GCs were enumerated in formalin-fixed paraffin embedded [FFPE] spleen sections using IHC); (C) splenic marginal zone B cells (triple immunofluoresence staining of spleen with anti-CD4 (Alexa Fluor 5646, red), anti-Metallophilic Macrophages 1 (MOMA1) (FITC, green), anti-IgM (Aminomethylcoumarin [AMCA], blue) was performed on frozen spleen sections; arrows/+ signs refer to areas of $\mathrm{IgM}^{+}$marginal zone $B$ cells adjacent to and extending outwardly beyond the MOMA ${ }^{+}$macrophage boundary) (20x magnification); and (H) splenic B220+B cells (B cells were enumerated in FFPE spleen sections using IHC). (I) Serum anti-nuclear antibodies (ANA) Abs were assessed after 7-week treatment for the groups indicated. Statistics indicate $(\mathbf{A}-\mathbf{C}){ }^{*} P<0.01,{ }^{*} P<0.0001$, G-744 versus vehicle; ${ }^{*} P<0.05$, Cyclophosphamide versus vehicle; " $P<0.05$, P505-15 versus vehicle; \& $P<0.05$, BR3-Fc versus Ig control; \$, \$, $P<0.01$, G-744 versus BR3-Fc, P505-15, or Cyclophosphamide, respectively. Groups were compared using (A and B) Log-rank Mantel-Cox test or (C) Kruskal Wallis test with Dunn's correction; (D-F, H, I) Group means \pm SEMs were calculated for each group; ${ }^{*} P<0.05$, ${ }^{*} P<0.01,{ }^{* *} P<0.005$, quantitative group means were compared with vehicle using (D-F, I) one-way ANOVA with Dunnett's correction or (H) 2-tailed heteroscedastic $t$ tests; symbols represent individual mice.

BCR activation in vitro was inhibited with similar potency (Table 2) and was significantly decreased in vivo compared with vehicle at concentrations of $10 \mathrm{mg} / \mathrm{kg}$ and above (Figure 1G). Furthermore, Btk inhibition abolished anti-CD40-stimulated B cell proliferation (Table 2). Syk inhibition with P505-15 blocked S473 Akt phosphorylation with similar potency as preventing B cell proliferation, consistent with a nonredundant 
Table 1. Potency and selectivity profile of G-744 in enzymatic assays

\begin{tabular}{lccccccccc}
\hline & BTK & BMX & ErbB2 & EphA7 & FGR & LCK & SLK & SRC & YES1 \\
$\mathbf{I C}_{\mathbf{5 0}}(\mathbf{n M})$ at $\boldsymbol{K}_{\mathbf{m}}$ app & 1.28 & $>3,333$ & 1,750 & 548 & $>1,111$ & $>3,333$ & $>10,000$ & $>3,333$ & $>10,000$ \\
Selectivity (fold) & 1 & $>2,604$ & 1,367 & 428 & $>868$ & $>2,604$ & $>7,813$ & $>2,604$ & $>7,813$
\end{tabular}

BMX, BM X kinase; ErbB2, epidermal growth factor receptor tyrosine kinase 2 (Her2); EphA7, Ephrin tyrosine kinase receptor A; FGR, Src-family tyrosine kinase Fgr; LCK, Iymphocyte-specific tyrosine kinase; SLK, STE20-like protein kinase; SRC, c-sarcoma virus tyrosine kinase; YES1, Yamaguchi sarcoma viral (v-yes) oncogene homolog 1 kinase; $K_{\mathrm{m}}^{\text {app }}$, apparent $K_{\mathrm{m}}$.

role of Syk in Akt phosphorylation (Table 2). Conversely, Syk blockade demonstrated a minimal inhibitory effect on Btk-Y223 autophosphorylation. As expected, Syk was essential for PLC $\gamma$ activation, as P505-15 treatment abrogated BCR-induced $\mathrm{Ca}^{2+}$ signaling in a dose-dependent manner (Figure 1E). These data indicate that both Syk and Btk play an essential role in triggering $\mathrm{Ca}^{2+}$ flux downstream of the BCR. Interestingly, Syk inhibition had a lesser effect on CD40-mediated murine B cell proliferation (Table 2), indicating that BCR and CD40 signaling pathways may converge at the level of Btk but not Syk in mice.

We next assessed whether specific Btk blockade with G-744 impacts BR3 signaling. G-744 had minimal effect on BAFF-induced B cell survival in vitro and did not alter NFאB p100 processing to p52 (Supplemental Figure 1, A and B). Similarly, 1-week in vivo treatment with G-744 did not alter follicular B cell numbers, although it partially reduced splenic marginal zone (MZ) B cell numbers (Supplemental Figure 1, C-E). In contrast, as expected, BR3-Fc treatment significantly reduced both follicular and MZ B cell numbers, while Syk inhibition most significantly lowered newly formed B cell numbers (Supplemental Figure 1, C-E). These data are in agreement with previously published data (35-37) that Btk and Syk are not major constituent of the BR3 survival pathway in follicular B cells and that blockade of BAFF and Btk are likely to have divergent effects on autoimmune responses due to their differential impact on B cell signaling and downstream functions.

As expected from the previously reported role of Syk and Btk in FcR function $(18,21,33)$, both Syk and Btk inhibition prevented Fc $\gamma$ RIII-triggered TNF $\alpha$ production with similar potency as BCR activation and proliferation (Table 2). These data demonstrate that G-744 is a highly selective Btk inhibitor that prevents both B cell BCR and macrophage Fc $\gamma$ R signaling with similar efficiency. Furthermore, our data indicate that Btk exhibits differential pathway activity in B cells compared with that reported for Syk inhibitors $(21,33)$.

Btk inhibition is superior to BAFF blockade or Syk inhibition in preventing IFNo-accelerated lupus and renal pathology. SLE patients often exhibit higher IFN $\alpha$ levels and type-I IFN activity that are associated with severe disease involving the kidneys, hematopoietic cells, and/or the CNS (38). We examined the efficacy of Btk blockade in preventing LN in the presence of increased levels of IFN $\alpha$ and highly aggressive clinical disease in NZB/W_F1 mice treated with adenovirus-expressing murine $\operatorname{IFN} \alpha(13,14)$ and compared it with BAFF blockade, Syk inhibition, and CTX. Mice treated with Ig control starting 3 weeks after adenovirus expressing IFN $\alpha(\mathrm{AdIFN} \alpha)$ injection succumbed to disease 9 weeks after AdIFN $\alpha$ administration. Btk inhibition with $100 \mathrm{mg} / \mathrm{kg} \mathrm{G}$-744 significantly improved overall survival (Figure 2A) and progression-free survival (Figure 2B), and stabilized proteinuria (Figure 2C) compared with vehicle. Analyses of drug concentration required to achieve inhibition of pBtk in NZB/W_F1 whole blood in vitro and actual G-744

Table 2. Potency of G-744 and P505-15 in murine and human cell-based assays

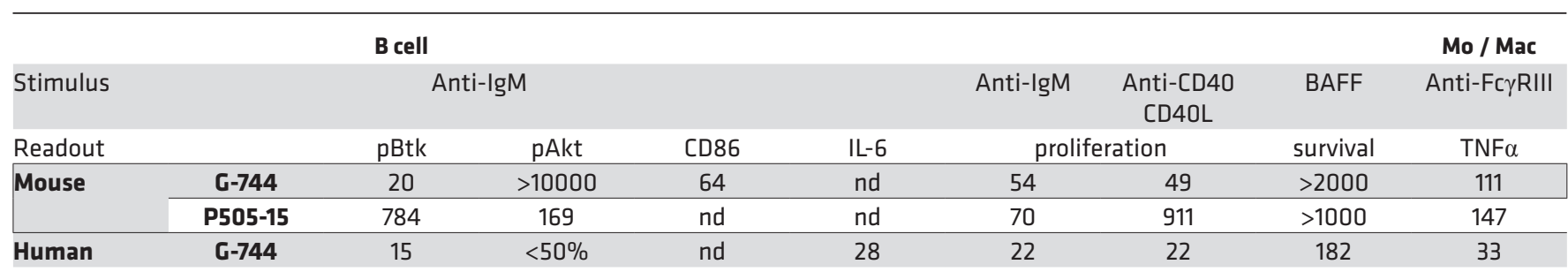

Values given are mean of the $\mathrm{IC}_{50}$ values $(\mathrm{nM})$ for $\mathrm{B}$ cell and macrophage assays with the indicated stimulation conditions; nd, not determined. 

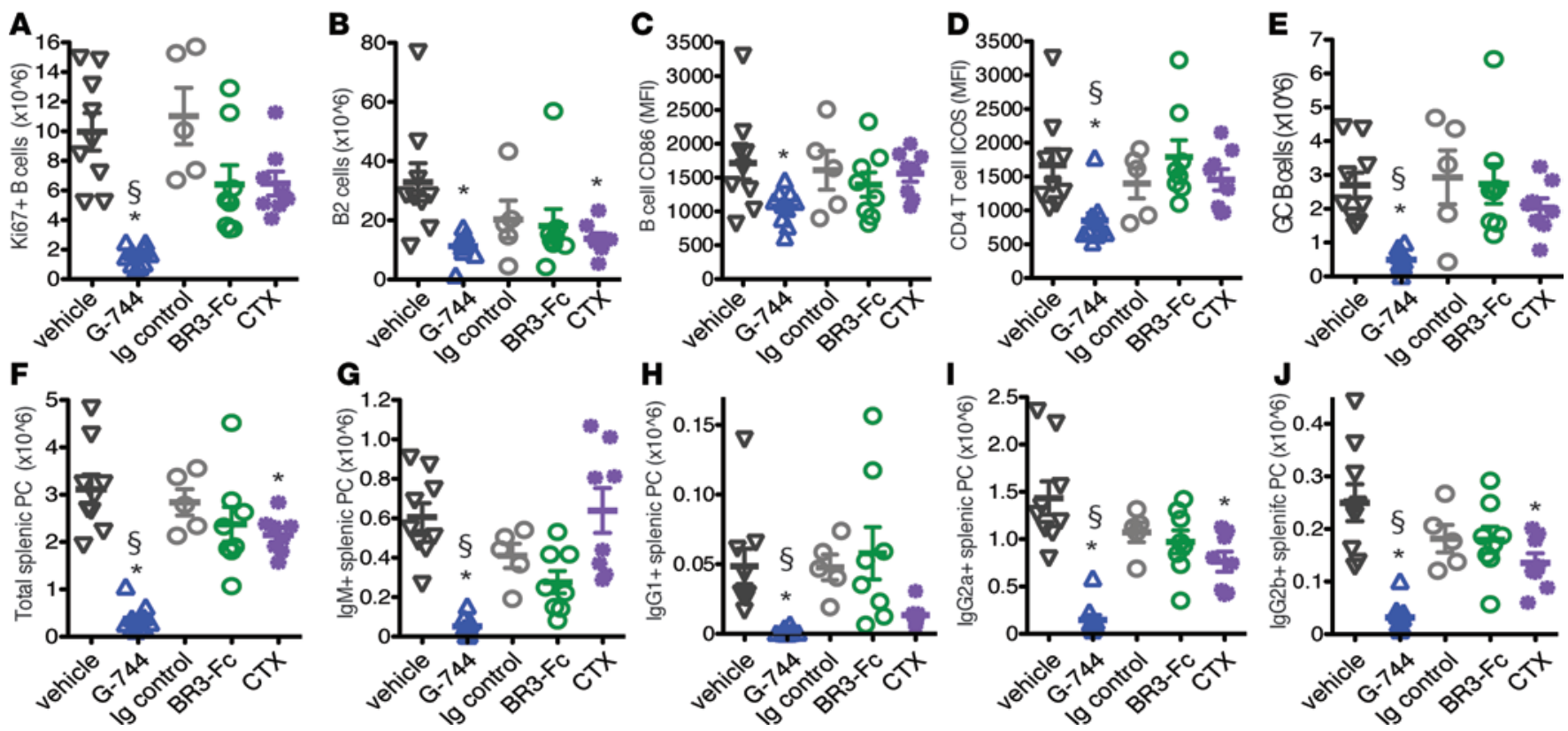

Figure 3. Effect of G-744 on splenic B, T, and plasma cells in IFN $\boldsymbol{\alpha}$-accelerated NZB/W_F1 lupus model. Fourteen-week-old preautoimmune NZB/W_F1 female mice ( $n=10$ /group) were administered with adenovirus containing murine IFN $\alpha$. Drug treatment was started 3 weeks after AdIFN $\alpha$ injection with $100 \mathrm{mg} / \mathrm{kg}$ G-744, vehicle HPMC, $5 \mathrm{mg} / \mathrm{kg}$ BR3-Fc, $5 \mathrm{mg} / \mathrm{kg}$ Ig control Abs, or Cyclophosphamide (CTX) as described in Methods and continued for 4 weeks. Graphs show the number and phenotype of splenic lymphocyte population from individual surviving mice as determined by flow cytometry and expressed as mean \pm SEM, symbols represent individual mice: (A) number of proliferating B cells (CD5-B220+Ki67+); (B) number of B2 cells (B220+CD38 $\left.{ }^{+}\right)$; (C) CD86

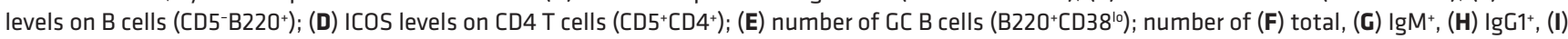
IgC2 $\mathrm{a}^{+}$, and (J) IgC2 $\mathrm{b}^{+}$splenic CD138+B220-/10 PCs. Significance indicate ${ }^{*} \mathrm{P}<0.05$, G-744 or CTX versus vehicle, BR3-Fc versus Ig control (one-way ANOVA with Dunnett's multiple comparison test); ${ }^{\circledR} P<0.05$, compare G-744 with BR3-Fc (2-tailed Student's $t$ test with Welch's correction).

plasma drug concentrations from the animals in the pharmacokinetic study group demonstrated that drug levels that were at least $70 \%$ inhibitory for pBtk were maintained throughout the dosing regimen (Supplemental Figure 2, A and C). In comparison, a similar extent of Syk inhibition at $60 \mathrm{mg} / \mathrm{kg}$ P505-15 (based on P505-15 concentration required to suppress FceRI-induced CD63 levels on basophils in whole blood, Supplemental Figure 2B and ref. 32) significantly improved progression-free survival (Figure 2B) but only showed a trend of ameliorating overall survival compared with vehicle $(P=0.2$; Figure $2 \mathrm{~A})$ and did not prevent progression of proteinuria (Figure $2 \mathrm{C}$ ). BR3-Fc showed a significant effect on overall survival but not on progression-free survival $(P=0.4)$ or proteinuria. CTX improved overall survival, progression-free survival, and proteinuria. Interestingly, Btk inhibition at $100 \mathrm{mg} / \mathrm{kg}$ showed markedly increased overall survival, compared with BR3-Fc, and progression-free survival, compared with all 3 alternate treatments (P505-15, BR3-Fc, and CTX). Furthermore, G-744 was superior in preventing proteinuria compared with P505-15 and BR3-Fc. At the end of the study, G-744 showed a trend in improving severity of glomerulopathy and significantly suppressed arteritis/periarteritis compared with surviving mice in the vehicle group with a relative effect at least comparable with that of CTX. Of note - in an alternate IFN $\alpha$-accelerated lupus study in which NZB/W_F1 mice were treated for only 4 weeks with G-744, BR3-Fc, or CTX to allow for a greater number of surviving mice in the control groups for comparison - G-744 treatment significantly prevented both glomerulopathy and artheritis/periarteritis compared with vehicle and glomerulopathy compared with BR3-Fc (Supplemental Figure 3, A-D). Syk inhibition did not significantly improve renal histopathology parameters (Figure 2, D and E).

Btk inhibition reduces GCS and Ab production in both IFNa-accelerated and spontaneous lupus. To assess the effects of sustained drug treatment on splenic B cell populations, we examined spleen sections in surviving mice from the IFN $\alpha$-accelerated lupus study using IHC or immunofluorescence. Treatment with G-744 significantly decreased GC frequency by $60 \%$ compared with vehicle, while BR3-Fc, CTX, and P505-15 exhibited only a minimal effect in suppressing splenic GC formation (Figure 2F). In contrast, BR3-Fc treatment resulted in loss of MZ B cells (Figure 2G), with G-744, P505-15, or CTX having no discernible effect on splenic MZ morphology. BR3-Fc treatment also decreased the number of splenic $\mathrm{B} 220^{+} \mathrm{B}$ cells as deter- 
A

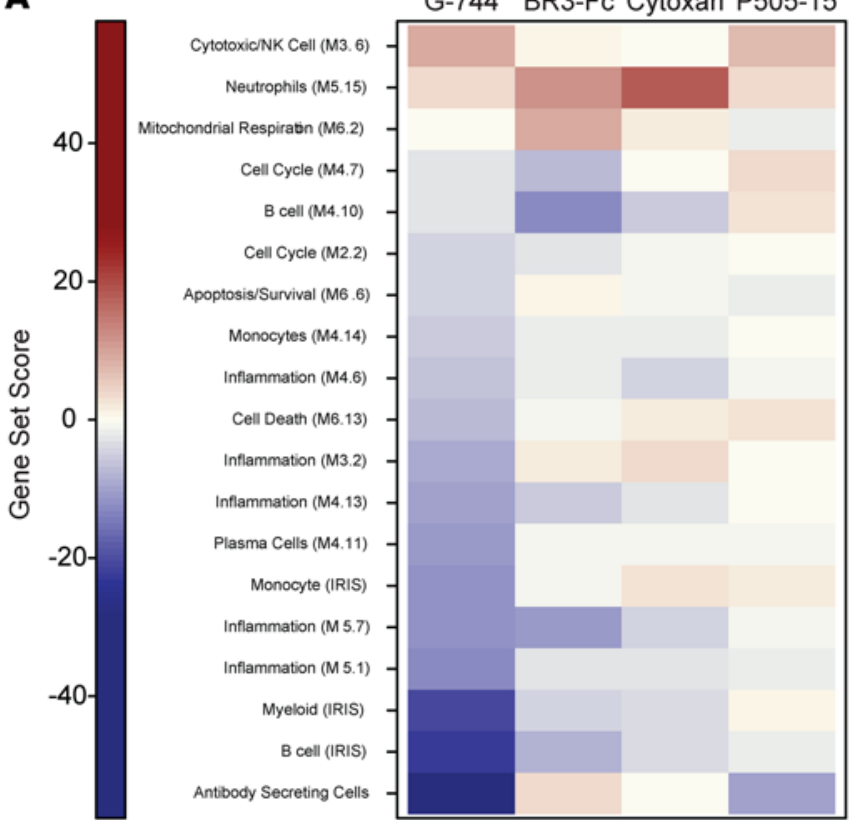

B

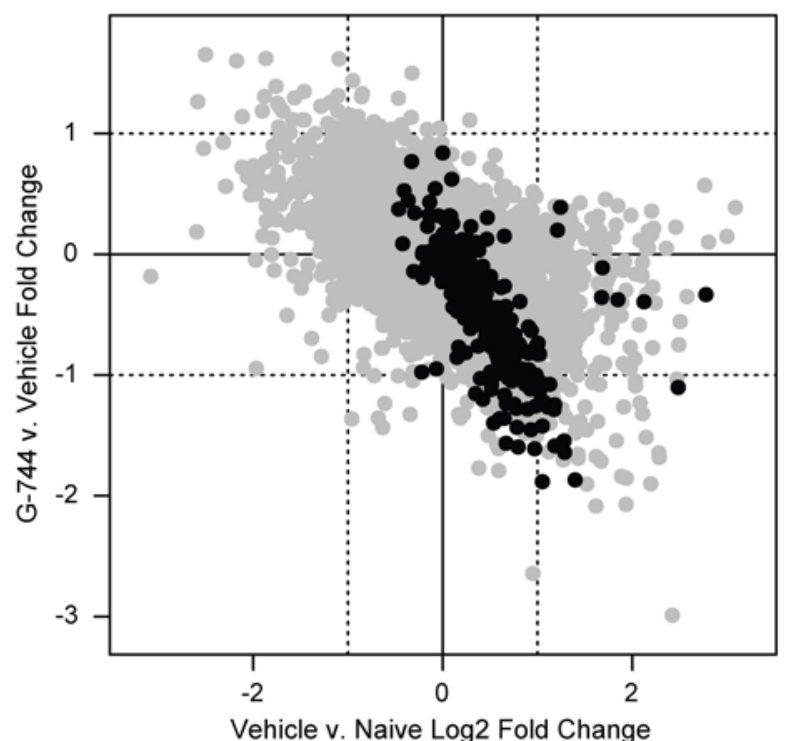

C

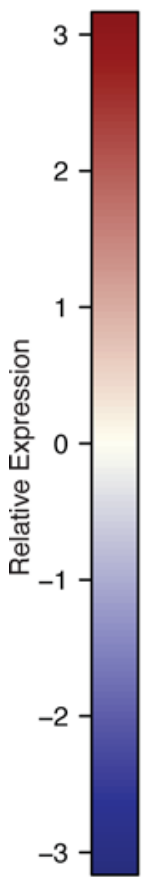

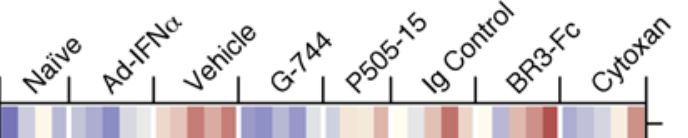$$
\text { Fos }
$$

Entpd1
Tigit Tram2

Sec24a

Cpeb2

Enpp1

Txndc11 -

Bhlha15

Edem1

Xbp1

Fkbp11

Creld2 -

Sdf2l1 -

Pon3 -

Eaf2 -

Rexo2 I830127L07Rik

Bst2

Ly6c1

Ly6c2

Zbp1

Mnda

Serpina3f

Spon1
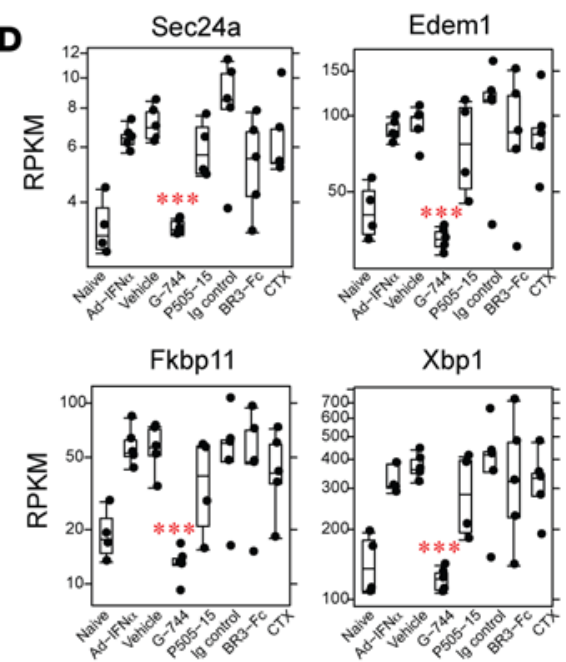

E

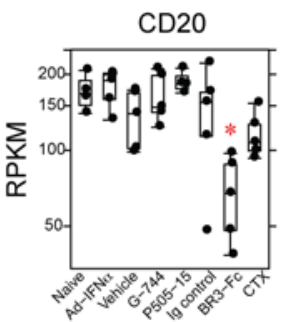

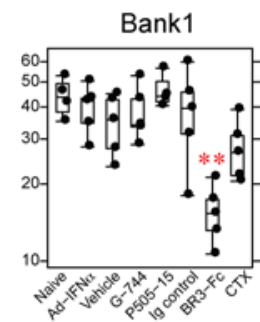

Figure 4. Gene expression changes in spleen after cyclophosphamide treatment or blockade of Btk, Syk, or BAFF in IFN $\alpha$-precipitated LN in NZB/W_F1 mice. LN was IFN $\alpha$-accelerated in female NZB/W_F1 mice as described in Figure 3. Mice were treated for 4 weeks starting 3 weeks after AdIFN $\alpha$ induction with vehicle, $100 \mathrm{mg} / \mathrm{kg}$ G-744, $60 \mathrm{mg} / \mathrm{kg}$ P505-15, 7.5mg/kg lg control, $7.5 \mathrm{mg} / \mathrm{kg} \mathrm{BR3-Fc,} \mathrm{or} \mathrm{Cyclophosphamide} \mathrm{(Cytoxan/CTX)} \mathrm{(} n=$ 10/group), followed by RNA sequencing analysis of splenic RNA. Two groups of 17-week-old NZB/W_F1 animals were also included (see Supplemental Figure 7) that were either not injected with AdIFN $\alpha$ (naive group) or treated for 3 weeks with AdIFN $\alpha$ (AdIFN $\alpha$ group). (A) Gene set enrichment analysis was performed using predefined immune gene modules ( $n=5$ /group). Gene sets that showed significant enrichment (permutation $P<0.01$ ) are shown as a heatmap indicating the magnitude of the gene set enrichment statistic. (B-D) G-744 reduces transcripts associated with ASC generation. (B) Calculated fold-changes in spleen from vehicle-treated versus naive animals ( $x$-axis), or $G$-744-treated versus vehicle-treated animals ( $y$-axis), are shown for all transcripts (gray) or ASC-specific transcripts (black). Dotted lines indicate a 2-fold change in expression. (C) Heatmap visualization of select ASC-specific transcripts that were induced (log2-fold change $>2, P<0.05$ ) in spleens of vehicle-treated animals compared with naive animals. Each column in the heatmap represents 1 animal within each treatment group. Genes marked in blue were reduced after G-744 treatment compared with 
vehicle-treated animals (fold change $>1.5, P<0.05$ ). ( $\mathbf{D}$ and $\mathbf{E}$ ) Box plot of reads per kilobase of transcript per million mapped reads (RPKM) expression of (D) select individual genes from C significantly affected by G-744 treatment and (E) select B cell genes affected by BR3-Fc treatment. Gene expression data were plotted using box plots overlaid with all observed data points. The box corresponds to the 1st quartile, median (black line), and 3rd quartile. The whiskers extend to the most extreme data point that is no more than 1.5 times the interquartile range from the box; ${ }^{*} P<0.05$, ${ }^{* *} P<$ $0.005,{ }^{* *} P<0.0005$, using DESeq2 to fit a negative binomial model, as described above.

mined by evaluating the $\mathrm{B} 220^{+}$immunoreactivity in spleen sections (Figure $2 \mathrm{H}$ ). These findings are consistent with the role of BAFF in B cell survival. In comparison, CTX and P505-15 treatment showed a modest effect and G-744 a minimal effect on $\mathrm{B} 220^{+}$reactivity. Examination of serum autoantibodies at 7-week treatment showed that ANA Abs were significantly reduced by G-744, but not by the other treatments (Figure 2I). Anti-dsDNA Abs showed a trend of reduction by G-744 for IgM isotypes (Supplemental Figure 4A) but no significant effect for IgG3-specific anti-dsDNA Abs (Supplemental Figure 4B). In comparison, BR3-Fc significantly reduced IgG3 anti-dsDNA Abs. Similar results were obtained in an alternate study where we compared Btk inhibition at $35 \mathrm{mg} / \mathrm{kg}$ or $100 \mathrm{mg} / \mathrm{kg}$ of G-744 to that of BAFF blockade or CTX treatment in IFN $\alpha$-accelerated NZB/W_F1 lupus (Supplemental Figure 5, A-G). We further analyzed total serum Ig concentrations in this model and observed that G-744 reduced titers of IgM and IgG3 Abs (Supplemental Figure 4, C and F) but did not affect titers of IgG1 or IgG2a (Supplemental Figure 4, D and E).

Given previous reports that Btk inhibitor treatment in spontaneous lupus disease in NZB/W_F1 mice without IFN $\alpha$-acceleration reduced IgG dsDNA Ab titers $(23,24)$, we also examined the effect of highly selective Btk inhibition with G-744 on disease parameters and autoantibody titers in NZB/W_F1 mice with spontaneously occurring disease and compared it to BR3-Fc and CTX. Therapeutic treatment with G-744 at $35 \mathrm{mg} / \mathrm{kg}$ and $100 \mathrm{mg} / \mathrm{kg}$ in contrast to BR3-Fc significantly improved overall survival (Supplemental Figure 6A) and progression-free survival (Supplemental Figure 6B), and it stabilized proteinuria (Supplemental Figure 6C); it also showed a significant dose-responsive inhibitory effect of $100 \mathrm{mg} / \mathrm{kg}$ G-744 treatment on GC formation compared with CTX (Supplemental Figure 6D). Surprisingly, in contrast to previously published data, BR3-Fc in this study did not prolong survival, perhaps due to the late treatment start at 36 weeks of age and the highly advanced proteinuria in this cohort. G-744 at $100 \mathrm{mg} / \mathrm{kg}$, but not BR3-Fc or CTX, significantly reduced serum anti-dsDNA (Supplemental Figure 6E) and anti-histone (Supplemental Figure 6F) autoantibody levels after treatment for 56 or 112 days. Furthermore, gene expression analyses from whole blood demonstrated that expression of B cell (Ighg1), myeloid cell (Csf1r), and IFN-regulated genes (Irf7, Oas3, Cmpk2, Mx1) were significantly decreased over time with G-744 treatment (Supplemental Figure 6G).

Taken together, these results indicate that highly selective Btk inhibition is superior to BAFF blockade, Syk inhibition, and CTX in ameliorating disease parameters and autoantibody production in the IFN $\alpha$-accelerated and spontaneous NZB/W_F1 lupus models.

G-744 significantly reduces splenic PCs. Given the effect of selective Btk antagonism on GC frequency and autoantibody production in therapeutic treatment regimens, we examined in more detail the mechanism of action of Btk inhibition with G-744 treatment on B cell subsets in spleen in the presence of elevated IFNo levels. Btk inhibition caused a potent decrease in the number of proliferating $\mathrm{Ki}^{+} \mathrm{B}$ cells compared with vehicle, BR3-Fc, and CTX-treated mice after 4 weeks of treatment (Figure 3A). Consistent with the effect on proliferating B cells, G-744 but not BR3-Fc reduced the number of total B2 cells (Figure 3B). Antagonism of Btk, but not the other treatments, decreased B cell CD86 and CD4+ $\mathrm{T}$ cell ICOS levels (Figure 3, $\mathrm{C}$ and $\mathrm{D}$ ), as well as the number of GC B cells (Figure $3 \mathrm{E}$ ) compared with control-treated mice. Importantly, G-744 significantly diminished the total number of splenic PCs compared with BR3-Fc and vehicle (Figure 3F). Furthermore, Btk inhibition reduced the number of splenic $\operatorname{IgM}^{+}$plasmablasts, as well as all isotype-switched PCs tested (IgG1 ${ }^{+}, \operatorname{IgG} 2 \mathrm{a}^{+}$, and IgG2 $\mathrm{b}^{+}$) (Figure $3, \mathrm{G}$ and $\mathrm{H}$ ). In comparison, BR3-Fc did not significantly lower the numbers of total, $\operatorname{IgM}^{+}$, or isotype-switched PCs compared with control Ig-treated mice. CTX moderately lowered the number of total, IgG2 $\mathrm{a}^{+}$, and IgG $2 \mathrm{~b}^{+}$PCs and significantly reduced $\operatorname{IgG} 1^{+} \mathrm{PC}$ in the spleen (Figure 3, F and $\mathrm{G}$ ).

G-744 inhibits splenic gene expression associated with $B$ cell terminal differentiation. To investigate potential mechanisms by which above treatments influence LN and tissue homeostasis during disease progression, we performed transcriptional profiling by RNA sequencing of whole spleen RNA isolated from AdIFN $\alpha$-injected NZB/W_F1 mice after 4-week treatment with G-744, P505-15, BR3-Fc, CTX, or controls. Drug treatment started 3 weeks after AdIFNa injection and onset of proteinuria when 
A

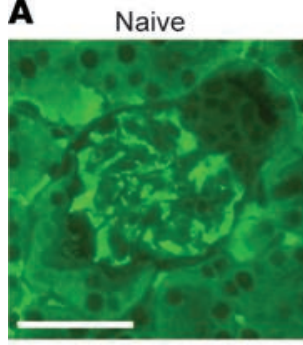

G-744

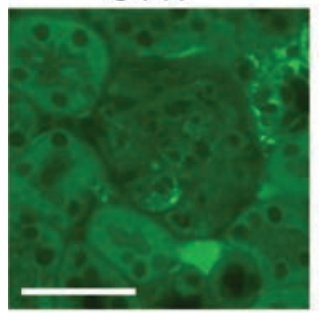

Ig Control

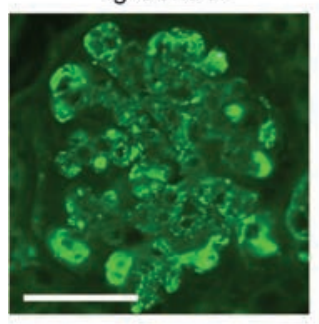

Vehicle

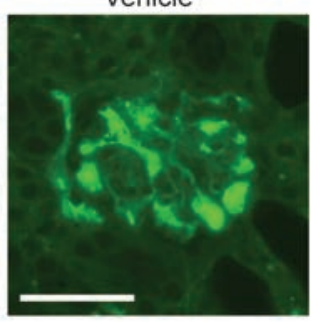

P505-15

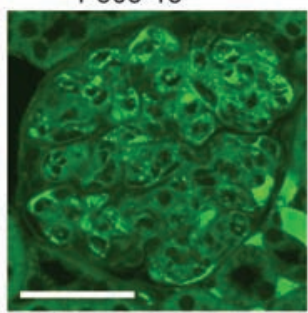

BR3-Fc

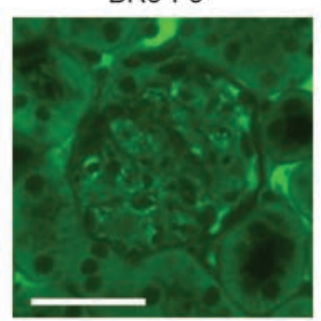

CTX

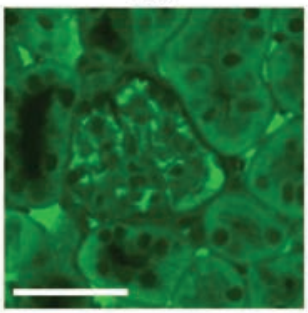

B

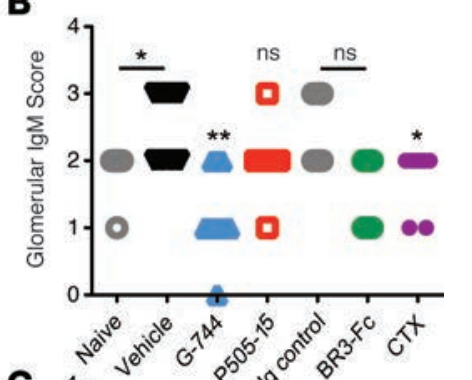

C

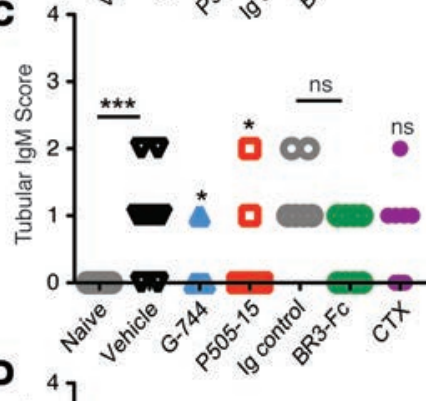

,
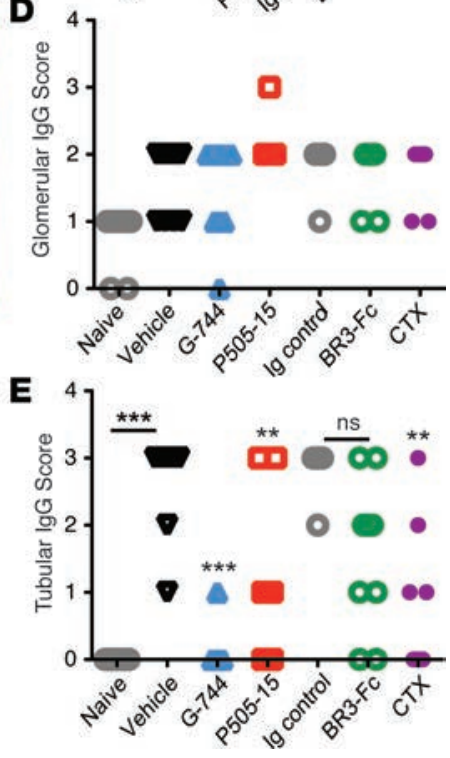

Figure 5. Effect on renal IgM and IgC deposition after short-term treatment with G-744, P505-15, or cyclophosphamide in IFN $\alpha$-accelerated $\mathbf{L N}$. Kidney sections were examined for IgM and IgG staining for each group derived from the experiment in Figure 4. (A) Representative immunofluorescence images of kidney sections showing IgM-rich deposits in glomeruli. Images are magnified 100x. Scale bars: $50 \mu \mathrm{m}$. (B-E) Intensity scores of the (B) glomerular IgM signal (representative images shown in A), (C) tubular IgM signal, (D) glomerular IgC signal, and (E) tubular IgC signal from kidney sections for the groups indicated ( $n=10$, except Ig control [ $n=6]$, BR3-Fc $[n=8]$ ). Statistical significance was determined using Fisher's exact test between naive and vehicle groups; between vehicle and G-744, P505-15, and cyclophosphamide groups; and between Ig control and BR3Fc groups $\left({ }^{*} P<0.05,{ }^{* *} P<0.005,{ }^{* *} P<0.0005\right)$.

mice were 17 weeks old (proteinuria $\leq 100 \mathrm{mg}$ / dl, Supplemental Figure 7A). AdIFN $\alpha$-treated 21-week-old NZB/W_F1 mice in the vehicle or Ig control group at the end of the study were nephritic (at least $80 \%$ of mice with proteinuria $\geq 300 \mathrm{mg} / \mathrm{dl}$ ), whereas all drug treatment partially prevented progression of proteinuria. For comparison, we included a group of 17-weekold NZB/W_F1 animals that were not injected with AdIFN $\alpha$ showing no or minimal proteinuria (termed naive group) and a group of 17-week-old mice that were terminated after 3-week AdIFN $\alpha$ induction (AdIFN $\alpha$ group).

Gene set enrichment analysis (GSEA) of spleen using gene sets describing antibody-secreting cells (ASC), other immune cells, inflammation, and general repertoire modules (39-41) demonstrated that G-744 treatment decreased gene expression associated with inflammation and cell death in the spleen (Figure 4A). Consistent with our in vivo findings, there was a strong inhibitory effect of G-744 treatment on modular repertoires associated with immune cell types including B cells, PCs, myeloid cells, and monocytes in the spleen (Figure 4A). Of particular note, the gene set that had the strongest inhibition due to Btk inhibition was the ASC set (Figure 4, A-D) that was recently defined (41). In comparison, other treatments did not result in decreases of equal magnitude. BR3-Fc treatment had moderate effects on select predefined B cell and cell cycle modules, but not ASCs. Specifically, BR3-Fc downmodulated expression of B cell lineage marker genes in the "B cell M4.10 module" including Cd19, Cd79a, Blk, Bank1, and Ms4a1 (Cd20), in accordance with its role in B cell survival (Figure 4E). Few changes in gene expression were observed in CTX or Syk inhibitor treatment, except upregulation of a neutrophil gene set by CTX and a mild decrease in ASC-associated gene expression after Syk inhibition (Figure 4A).

Examination of changes in individual ASC genes revealed that the majority of these genes were induced in animals with disease as compared with naive controls (Figure 4B, indicated by the back dots), and expression of these same genes was downmodulated by G-744 treatment. Further analyses of these ASC-specific transcripts that were induced (fold change $>2, P<0.05$ ) in vehicle-treated compared with naive animals showed that G-744 normalized expression of the majority of them, while Syk inhibition, BAFF blockade, and CTX exhibited little effect (Figure 4C). These genes, including Sec24a, Edem1, Fkbp11, and Xbp1 (Figure 4, C and D), encompassed biological function connected with Ab secretion such as the unfolded protein response, protein post-translational modifications, folding, and transport in the ER. 
A

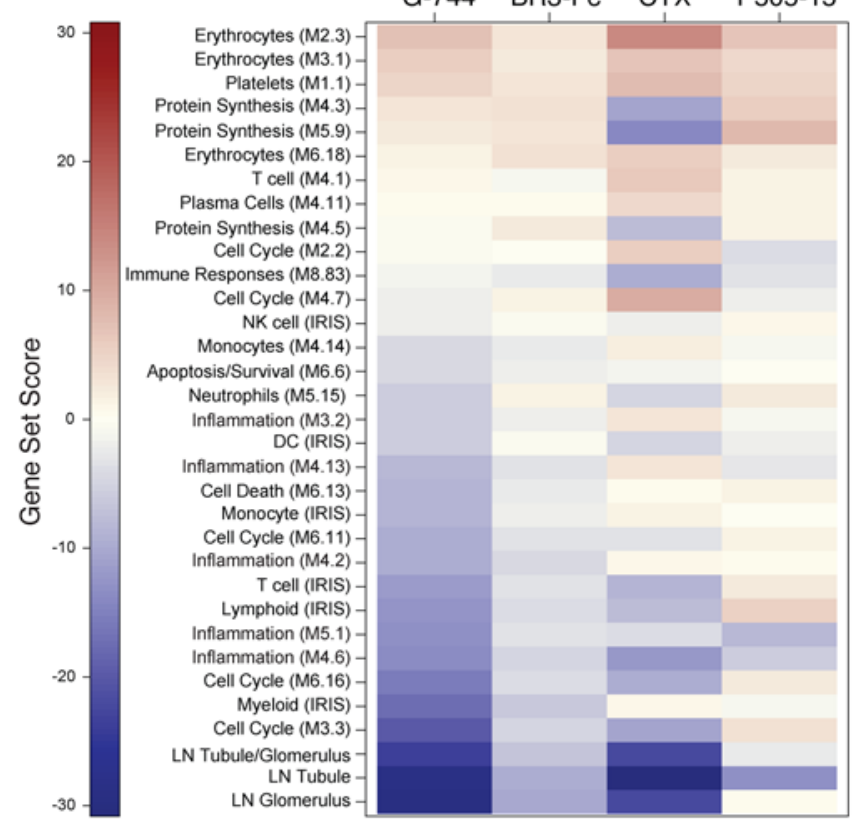

B

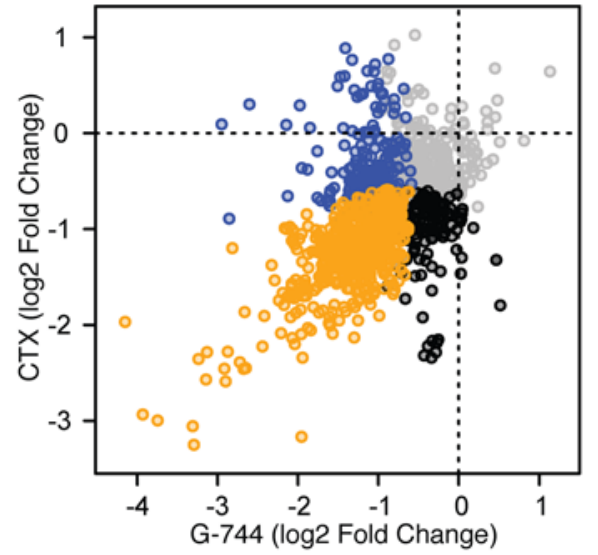

C

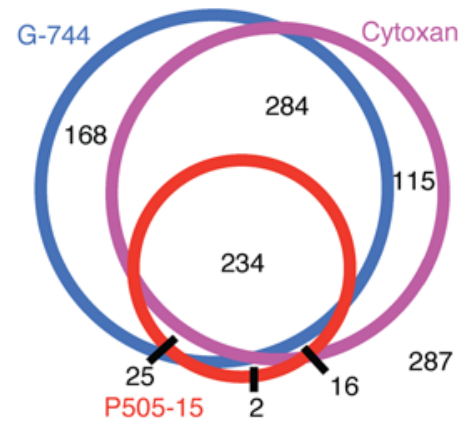

D

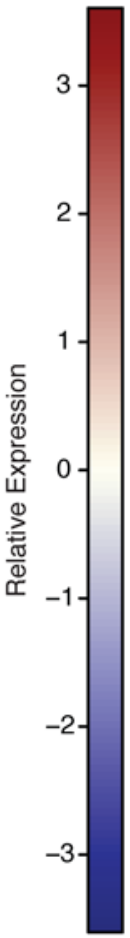

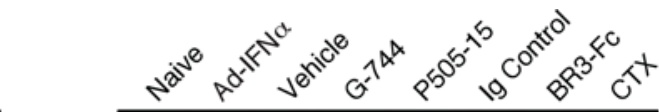
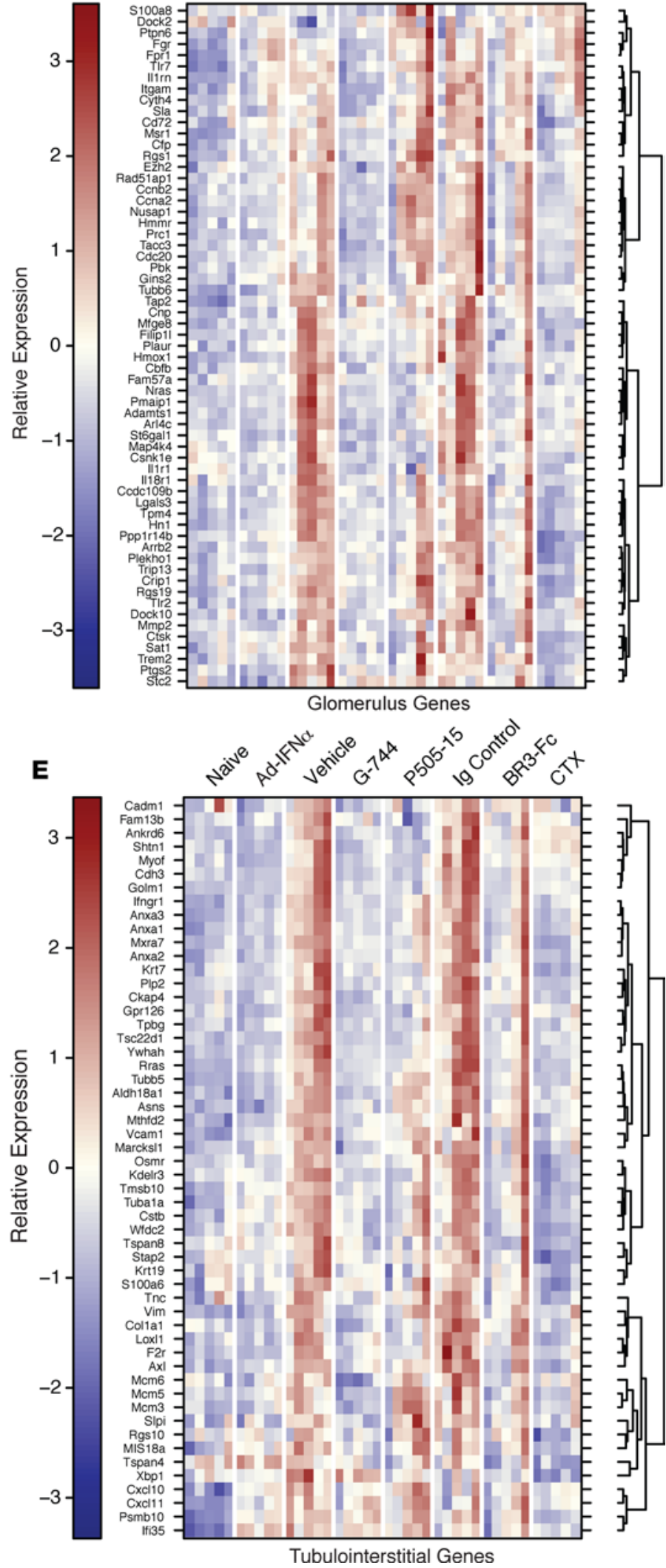

Figure 6. Gene expression changes in kidney in IFN $\alpha$-accelerated LN in NZB/W_F1 mice after treatment with G-744, P505-15, BR3-Fc, or cyclophosphamide (Cytoxan). RNA sequencing analysis was performed on kidney RNA ( $n=5$ /group) derived from the experiment described in Figure 4. (A) Gene set 
enrichment analysis of predefined immune (see Figure 4A) and human LN ortholog gene modules (as defined in $\mathbf{D}$ and $\mathbf{E}$ ); gene sets that showed significant enrichment (permutation $P<0.01$ ) are shown as a heatmap indicating the magnitude of the gene set enrichment statistic. (B and $\mathbf{C})$ Analyses of overlapping effects on renal gene transcription by drug treatments. (B) Renal transcripts that were significantly upregulated in diseased kidneys versus naive animals (2-fold elevated with FDR of 0.05) were assessed for their downregulation ( 21.5 -fold with FDR of 0.05) by cyclophosphamide (CTX) versus G-744 treatment. Blue dots indicate genes significantly reduced by G-744, black dots indicate genes significantly reduced by CTX, and orange dots indicate genes significantly downregulated by both treatments. (C) Venn diagram showing the degree of overlap of genes upregulated in the kidneys of diseased animals that were subsequently decreased by CTX, G-744, or P505-15 treatment using the same statistical cutoffs as stated for B. The number of genes affected by each treatment is indicated within each intersection, and the number of genes unaffected by any of these treatments is indicated outside of the circles. ( $\mathbf{D}$ and E) Effect of drug treatment on human LN ortholog genes previously identified to be upregulated in the (D) glomerulus or (E) tubulointerstitium of nephritic kidneys from human LN patients (see Methods). Genes that showed individual significant changes with any treatment versus their controls are indicated ( $\geq 1.5$-fold difference between groups with an adjusted $P<0.01$ as determined using a negative binomial generalized linear model). Each column in the heatmap represents 1 animal within each treatment group.

G-744 treatment reduces IgM and IgG deposition in kidney glomeruli and proximal convoluted tubules. In light of the profound effects on ASC gene expression in the spleen of Btk inhibitor-treated NZB/W_F1 mice, we assessed the effect of specific Btk inhibition compared with Syk blockade and CTX on renal IgM and IgG deposition from the experiment described in Figure 4. Proteinuric vehicle-treated animals showed an increase in IgM staining in glomeruli (Figure 5A), and quantification of these deposits revealed a statistically significant increase in IgM-rich inclusions in both glomeruli and proximal convoluted tubules in diseased versus naive mice (Figure 5, B and C). In contrast, there was a much less pronounced increase in glomerular IgG deposition with disease progression compared with glomerular IgM and tubular IgG deposits (Figure 5, B, D, and E). G-744 treatment resulted in a significant reduction in both glomerular and tubular IgM deposits (Figure 5, B and C), while IgG deposits were significantly reduced only in the tubules (Figure 5, D and E). P505-15 treatment significantly reduced tubular but not glomerular IgM scores, whereas CTX reduced glomerular but not tubular IgM scores (Figure 5, B and C). Significant but lower magnitude decreases in tubular IgG scores were also observed in P505-15- and CTX-treated animals. In comparison, BR3-Fc treatment showed no significant decrease in tubular scores (Figure 5E). Further examination of IgG in the glomeruli showed that none of the drugs after 4 weeks of treatment significantly lowered glomerular IgG deposits (Figure 5D), although glomerulopathy was significantly reduced by G-744 treatment (Supplemental Figure 7B) as previously observed (Supplemental Figure 3B).

Btk inhibition and CTX treatment show overlapping effects in reducing renal gene expression linked to $L N$ in $N Z B / W \_F 1$ mice. To determine effects of drug treatment on LN in mouse kidneys, we performed transcriptomic profiling of whole kidney RNA from the study in Figure 4 using RNA sequencing coupled with GSEA analyses using inflammatory and general repertoire modules examined above for spleen (Figure 6A). G-744 treatment showed a pronounced effect on gene modules reflective of myeloid cell activation, cell cycle, and several inflammation repertoires, while Syk inhibition reduced gene transcription associated with only one of the inflammation modules. BAFF blockade had little effect on global renal gene transcription. CTX treatment decreased transcriptional modules linked to protein synthesis, select inflammatory patterns, and immune responses associated with antigen presentation - in particular H2, class-II MHC gene expression. Of note, CTX increased erythrocyte transcriptomes and displayed mixed effects on cell cycle modules that were either under- or overexpressed (Figure 6A).

Analyses of genes increased during IFN $\alpha$-accelerated nephritis and downregulated by more than one drug treatment revealed that Btk inhibition and CTX treatment demonstrated overlapping effects on decreasing disease-induced genes: of 1,131 upregulated transcripts ( $>2$-fold induced in vehicle versus naive group, with an adjusted $P<0.05$ ), 46\% of the transcripts (518 total) were downregulated by both G-744 or CTX treatment at least 1.5-fold, with an adjusted $P<0.05$ (Figure 6, B and C). In comparison, Syk inhibition only reduced a subset of the transcripts concomitantly decreased by G-744 or CTX (45\%, 234 total) and showed minimal unique overlap with CTX (H2 class-II MHC, CD74) or G-744 (PTPRN) (Figure 6C).

The most highly expressed genes during onset of clinical nephritis that were reduced either by G-744 or CTX were known mediators of tubulointerstitiaI damage or fibrosis (IL1-F6, Grem1, Lcn2) and tissue remodeling associated with myeloid cell recruitment and activation (Cxc117, Timp1). Unique nephritis-induced genes downregulated predominantly by Btk inhibition included myeloid cell receptor genes (Cec4d [MCL], Clec4n [Dectin-2], Itgam, Trem1), genes associated with B cell proliferation (Fcr15), and several transcription factor-encoding genes (Btg2, Egr1, Fos, Fosb, Irf4, Jun).

Btk inhibition reduces renal gene expression associated with $L N$ in human patients. To understand the possible relevance to human disease, we intersected genes previously shown to be elevated during nephritis in 
A

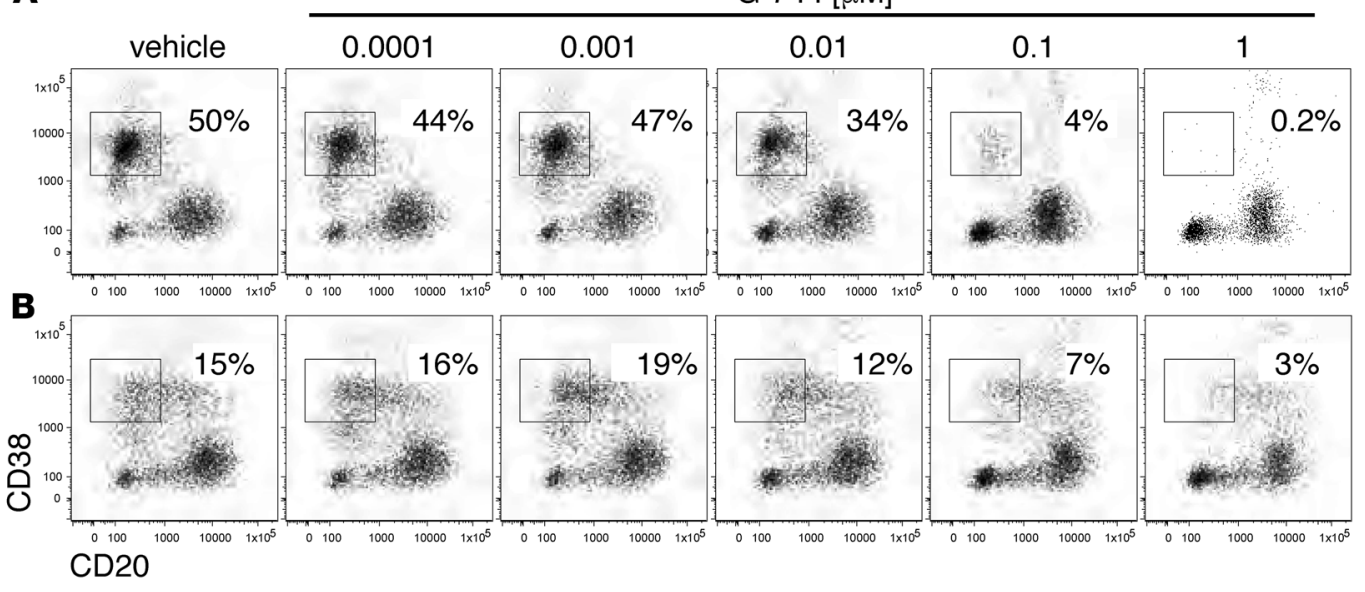

C

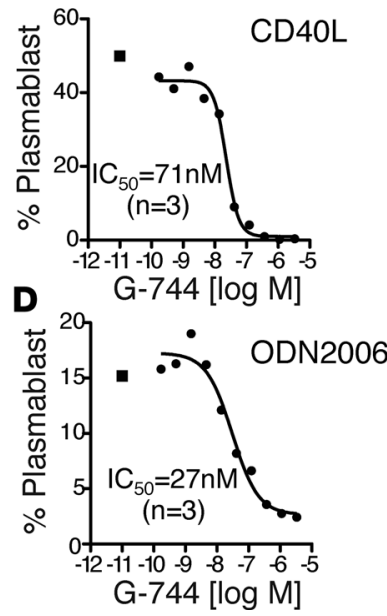

Figure 7. Btk blockade reduces differentiation of human memory B cells into plasmablasts in the presence of IFN $\alpha$. Peripheral blood human memory B cells (CD27 ${ }^{+}$CD19+) were purified and cultured for 7 days with sCD40L (A and C) or ODN2006 (B and D) plus IL-2, IL-6, IL-10, IL-15, and IFN $\alpha$, in the presence of vehicle or different concentration of G-744 as indicated. Percentage of plasmablasts (CD20-CD38++) was determined by flow cytometry. (A and B) Representative CD20/CD38 FACS profiles for individual treatments with vehicle or G-744 as indicated in response to (A) CD40L or (B) ODN2006 plus cytokines. Percentages of plasmablasts are shown in the graphs. (C and D) Dose-response curve of G-744 on plasmablast percentage after stimulation with (C) CD40L or (D) ODN2006 plus cytokines for the experiment in $\mathbf{A}$ or $\mathbf{B}$, respectively. One representative experiment of $\mathbf{3}$ independent experiments is shown.

kidneys isolated from different lupus mouse strains with genes upregulated in (i) the glomeruli, (ii) the tubulointerstitium, or (iii) both compartments from kidney biopsies of human LN patients versus normal control subjects (42) and assigned them to 3 modules (Figure 6A). Examination of drug treatment effects on gene expression associated with human $\mathrm{LN}$ in these renal compartments demonstrated that therapeutic treatment with G-744 significantly abrogated transcripts associated with both glomerular and tubular gene expression (Figure 6, D and E; and Supplemental Figure 8A). CTX treatment significantly inhibited the transcriptome of the tubulointerstitial compartment with a lesser effect on the glomerular module compared with G-744. In comparison with G-744 and CTX, BR3-Fc or P505-15 showed a minimal effect (Figure 6, D and E; and Supplemental Figure 8A). Assessment of individual genes defined by Berthier et al. for the glomerulus and the tubulointerstitial compartment (Figure 6, D and E) or genes common to both compartments (Supplemental Figure 8A) confirmed that the genes were elevated in animals with active disease (vehicle and Ig drug-control groups) versus animals with minimal disease (naive group) (42). Interestingly, while the AdIFN $\alpha$ group showed a similar extent of ASC-associated transcript induction compared with vehicle/Ig control groups (Figure 4C), nephritis-induced gene expression was not fully developed in the AdIFNa group compared with control groups, in agreement with relative disease activity exemplified by the higher proteinuria and glomerulopathy scores in the control groups (Supplemental Figure 7). Btk inhibitor-regulated genes expressed in the glomerulus only or in both compartments were enriched for genes encoding molecules associated with myeloid cells and inflammation (Cd14, Fgr1, Fpr1, IgSF6, IL1Rn, Msr1, Plaur, Sptlc2, Tlr1), cell cycle function (Ccnb2, Cdc20, Ckap2, Fam57a, Fam129a, Gins2, Nusap1, Prc1, Rad51ap1, Top2a), cell migration or adhesion (Ccr2, Cd44, Cxcr4, Hmmr [Cd168], Itgam [Cd11b], Lgals3, Rgs1), and extracellular matrix function and degradation (Adamts1, Mmp2, Col3a1, Timp1, Fn1) (Supplemental Figure 8, B and C).

Genes exclusively associated with tubule expression and inhibited by G-744 were enriched for additional molecules associated with extracellular matrix function and adhesion regulation (Cdh3, Colla1, CXCL10, Gpr126, Tpbg, Vcam1), as well as molecules that control membrane trafficking and may be associated with cell-extracellular matrix (ECM) interactions (Anxa1, Anxa2, Golm1, Kdelr3, Myof, Plp2, Slpi) (Supplemental Figure 8D). Several genes with less well-characterized functions - but with potential roles in cell proliferation, cell death, angiogenesis, or vascular function (Ankrd6, Anxa3, Asns, F2r11, Krt19,

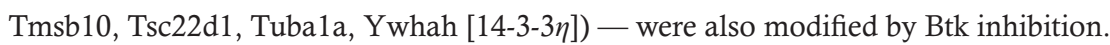

Therefore, short-term and highly specific inhibition of Btk in the NZB/W_F1 model affected both B cell and myeloid cell responses in the secondary lymphoid system and further decreased the expression of 
renal ortholog genes previously identified to be differentially expressed in the glomerular and tubulointerstitial compartments in human LN patients.

G-744 inhibits human $B$ cell activation and differentiation into plasmablasts. To examine whether effects observed with Btk inhibition in the mouse system translate to human, we assessed Btk pathway activity in human B cells and monocytes. In response to IgM activation, G-744 blocked pBtk with an $\mathrm{IC}_{50}$ of 15 $\mathrm{nM}$ (Table 2), while pAkt phosphorylation was not significantly changed. Furthermore, BCR-induced B cell proliferation and IL-6 production was inhibited with similar potency. B cell proliferation was also potently abrogated after CD40L stimulation. In comparison, BAFF-induced B cell survival was only partially reduced at G-744 concentrations that blocked BCR-triggered responses, albeit with a relatively higher impact of Btk inhibition on BAFF-mediated B cell survival in human B cells as compared with mouse B cells. As observed in murine B cells (Supplemental Figure 1B), Btk inhibition did not affect NFk-B2 p100 to p52 processing (data not shown). Finally, G-744 prevented Fc $\gamma$ RIII-mediated TNF $\alpha$ production in human monocytes (Table 2).

Given the reduction of PC generation by G-744 in NZB/W_F1 LN, we explored whether Btk contributes to TLR9-induced human memory B cell differentiation into plasmablasts. Stimulation of CD27 memory B cells with CD40L or TLR9 ligands in the presence of cytokines including IFN $\alpha$ (43) yielded about $50 \%$ or $15 \%$ plasmablast $\left(\mathrm{CD} 20^{-} \mathrm{CD} 38^{++}\right)$, respectively (Figure 7, A and B). G-744 potently abrogated both CD40L- (Figure 7, A and C) and ODN2006-induced (Figure 7, B and D) plasmablast generation in a dose-dependent manner. Importantly, we also observed that, while Btk is expressed in differentiated plasmablasts at a level comparable with naive B cells, inhibition with G-744 did not affect survival of plasmablasts and PCs in vitro (Supplemental Figure 9). These data demonstrate that Btk activity is essential for B cell proliferation and differentiation into plasmablasts in response to either CD40 or TLR9 activation in the presence of IFN $\alpha$, but it does not appear to be required for survival of these cells once they have differentiated.

\section{Discussion}

LN is a major cause of morbidity in SLE patients for whom no immune-based targeted therapies are currently approved $(15,16)$. We developed an oral highly specific reversible Btk inhibitor, G-744, with over 400-fold selectivity against all 284 kinases tested, including highest selectivity against Tec- and Src-family kinases compared with other Btk inhibitors (Supplemental Table 1). Specific Btk inhibition shows a profound effect on improving survival, as well as preventing glomerulonephritis and progression of proteinuria in animals with severe disease when high levels of IFN $\alpha$ are present in the IFN $\alpha$-accelerated (Figure 2) or the spontaneous NZB/W_F1 lupus model (Supplemental Figure 6). Notably, G-744 treatment is the most efficacious from all treatments tested and superior to CTX $(15,16)$, the BAFF antagonist BR3-Fc (17), and the Syk inhibitor P505-15 (32). Importantly, in NZB/W_F1 mice with IFN $\alpha$-accelerated lupus disease, our results demonstrate a global effect of specific Btk blockade on downmodulating both the transcriptome associated with PC generation in the spleen, in particular secretory pathway genes, as well as renal gene expression associated with LN in human SLE patients.

Interestingly, CTX and G-744 showed overlapping effects on inhibiting renal gene transcription associated with human LN, while CTX did not affect the PC program. BR3-Fc treatment, although moderately prolonging survival of mice, did not impact gene transcription associated with PC generation or nephritis, which is consistent with the modest disease-modifying effects observed in SLE patients. Surprisingly, Syk inhibition did not match Btk inhibition, and while P505-15 showed a similar trend in improving disease parameters compared with G-744 at drug levels that lead to comparable respective enzyme inhibition (Supplemental Figure 2), P505-15 treatment only minimally reduced gene transcription controlling $\mathrm{B}$ cell terminal differentiation and tubular damage. These results identify Btk as a gatekeeper of processes controlling glomerulonephritis in NZB/W_F1 mice with high levels of IFN $\alpha$ and provide insights that Btk-dependent pathways that drive disease in mice may also be active in human SLE.

IFN $\alpha$ triggers pleotropic effects on the immune system in both human and mice, including sustained B cell proliferation, upregulation of TLR7, activation of DCs and myeloid cells, induction of T-dependent immune responses, and impairment of GC selection resulting in increased autoreactivity of the antigen-driven $\mathrm{B}$ cell repertoire $(7,8,10,11,14)$. AdIFN $\alpha$ promotes a feed-forward amplification process in lupus-prone NZB/W_F1 mice triggering lupus disease that exhibits distinct features compared with the spontaneous model, including requirement of $\mathrm{T}$ cells for initiating disease, persistent production of short-lived PCs, enhanced 
GC reaction, and severe glomerular proliferative lesions with crescent formation $(5,14,44)$. Given these disease-promoting effects of IFN $\alpha$ in both human and mice, we chose to analyze the consequences of selective Btk inhibition in the AdIFN $\alpha$-accelerated NZB/W_F1 lupus model in particular, as this model is more resistant to therapeutic intervention, including CTX in combination with anti-CD40L and CTLA4-Ig (14).

Btk inhibition for 4 weeks at drug levels of G-744 that inhibit Btk enzyme activity throughout the dosing period ablated in the spleen the number of proliferating B cells, activated CD86 ${ }^{+} \mathrm{B}$ cells, GC B cells, and PCs (including $\operatorname{IgM}^{+}, \operatorname{IgG}^{+}, \operatorname{IgG}_{2} \mathrm{a}^{+}$, and $\mathrm{IgG} 2 \mathrm{~b}^{+} \mathrm{PCs}$ ), as well as ICOS ${ }^{+} \mathrm{CD} 4 \mathrm{~T}$ cells of AdIFN $\alpha$-treated NZB/W_F1 mice (Figure 3). In contrast, BR3-Fc treatment did not significantly affect splenic GC B cell or above $\mathrm{IgG}^{+} \mathrm{PC}$ populations, but it did show a trend in reducing proliferating $\mathrm{B}$ and $\mathrm{B} 2$ cells, while CTX had a modest effect on IgG2 $\mathrm{a}^{+}$and IgG2 $\mathrm{b}^{+}$PCs with little effect on B cell subsets. PCs were also reduced in the BM by Btk inhibition (data not shown), although — consistent with previous data — only low numbers of PCs were detected in the BM in vehicle-treated mice, in favor of large numbers of short-lived PCs in the spleen (14). Given the extensive effect on PC numbers, it is surprising that, while we did detect a significant reduction in ANA autoantibodies (including IgM, IgG, and IgA isotypes) measuring reactivity to various antigens (Sm, RNP, SSA/Ro, SSB/La, DNA, Scl70, and Jo-1), we did not observe a significant effect of G-744 after 6 weeks of treatment on anti-dsDNA IgG or IgG3 autoantibody titers in the presence of AdIFN $\alpha$ (Supplemental Figure 5E and Supplemental Figure 4B). However, anti-dsDNA IgM autoantibodies showed a trend of reduction $(P=0.077)$, and total IgM and IgG3 serum Ab concentrations were significantly reduced after 6 weeks of treatment with G-744 (Supplemental Figure 4 C-F), indicating a potent effect of G-744 treatment (i) on IgM production by short-lived plasmablasts that are predominant in this model or (ii) on alternate ANA Ab species. In comparison, anti-dsDNA and anti-histone Ab titers were significantly reduced with 100 $\mathrm{mg} / \mathrm{kg}$ G-744 in the spontaneous model after 6 weeks of treatment, in agreement with effects observed with less selective and/or irreversible Btk inhibitors in the spontaneous NXB/W_F1 model $(23,24)$ and in the BXSB-Yaa or the pristane DBA/1 lupus model that have a greater contribution of TLR7 and TLR7/type-I IFN pathways, respectively (29). Although unclear at present, the lack of G-744 effect in the IFN $\alpha$-accelerated model, specifically on dsDNA IgG autoantibody titers that are particularly dependent on IFN $\alpha / T L R 9$ signaling, may be due to the continued expression of high levels of exogenous adenoviral-derived IFN $\alpha$. In this respect, Btk inhibition reduced the peripheral blood IRG signature that accompanied severe disease onset around 36 weeks in the spontaneous model (Supplemental Figure 6G) but did not significantly impact the blood IRG signature in the IFN $\alpha$-accelerated model (data not shown). Priming B cells with IFN $\alpha$ lowers the BCR activation threshold and may trigger spontaneous peripheral activation of anti-DNA B cells in a TLR9-dependent manner and their differentiation into Ab-forming cells via an extrafollicular pathway (45, 46), which may be less dependent on BCR/Btk signaling and T cell help.

To further understand the mode of action of selective Btk inhibition in reducing PCs and GCs in spleen, genome-wide transcriptional profiling followed by GSEA identified a predominant role of Btk in controlling gene transcription associated with B cell terminal differentiation and ASCs, in addition to strong inhibitory effects on myeloid cells and monocyte modules. Btk inhibition resulted in downregulation of genes involved in most stages of the secretory process encoding molecules associated with folding of ER proteins, including ER chaperones (Fkbp11, Txndc11, Txndc5), degradation of misfolded ER proteins by the ERAD pathway (Edem1, -2, -3), protein glycosylation (Man1a, Manea, Fut8), and ER-Golgi vesicular trafficking (Sec24d, Sec24a). The main transcription factor that controls the secretory capacity of PCs is $\mathrm{XBP} 1$, and transcription of most of the above secretory pathway genes has been shown to be dependent on XBP1 (47). Accordingly, splenic XBP1 expression was also strongly inhibited by Btk inhibition and so were its regulators Ern1/IRE1 and Prdm1/Blimp1, as well as additional Blimp1-controlled genes (Sdc1, ELL2, IgJ) essential for PC terminal differentiation. Furthermore, G-744 inhibited IRF4 expression that, at higher doses, controls the initiation of PC differentiation. Downregulation of select splenic genes related to B cell differentiation, in particular Blimp1, have previously been noted in the spontaneous NZB/W_F1 model with RN486 (24), indicating that the transcriptional program related to B cell terminal differentiation may not be highly controlled by IFN $\alpha$. Unlike Btk inhibition, BAFF blockade had no effect on ASCs but had moderate effect on B cell marker- and cell cycle-associated gene expression, demonstrating that Btk inhibition and BR3-Fc exhibit divergent effects on B cell homeostasis. These data reveal Btk as a potential candidate to control an essential checkpoint in lupus B cell pathogenesis in the presence of IFN $\alpha$, as Btk blockade ablated most aspects of the PC program in NZB/W_F1 mice. In this respect, forced overexpression of Btk triggers increased PC numbers, anti-nucleosome autoantibody production, and SLE-like 
disease (48). Furthermore, suppression of PC generation may translate to humans, as Btk inhibition with G-744 prevented in vitro differentiation of human memory B cells to plasmablasts in response to CD40L or TLR9 ligands plus IFN $\alpha$.

The molecular mechanisms of how Btk regulates the PC program during terminal B cell differentiation are currently unknown, but Btk may exert its function due to its prominent role in $\mathrm{Ca}^{2+}$ homeostasis, controlling inositol trisphosphate generation and the depletion of thapsigargin-sensitive intracellular calcium stores in the ER, as well as sustained extracellular $\mathrm{Ca}^{2+}$ influx (49). Pronounced alterations in $\mathrm{B}$ cell activation thresholds due to signaling abnormalities and continued stimulation with autoantigens particularly after a break in B cell tolerance may lead to sustained BCR activation, as well as prolonged depletion of ER $\mathrm{Ca}^{2+}$ stores and extracellular $\mathrm{Ca}^{2+}$ influx in a Btk-dependent manner. Accordingly, peak BCR-triggered $\left[\mathrm{Ca}^{2+}\right]$ i responses were shown to be significantly higher in B cells from lupus patients compared with normal controls (50).

However, changes in $\mathrm{BCR}$ and $\mathrm{Ca}^{2+}$ signaling are likely only one component of many altering loss of $\mathrm{B}$ cell checkpoint function. Thus, deregulated CD40 pathway activity has been implicated in promoting pathogenesis in SLE (51), and Btk inhibition blocked anti-CD40-mediated B cell proliferation in murine and human B cells. In this respect, it is relevant to note that autoimmune pathology in CD19-hBtk mice is dependent on B-T cell interaction through CD40L and CD19-hBtk B cell-promoted ICOS T cell expression (52), consistent with our data demonstrating suppression of ICOS expression on CD4 T cells (Figure 3D). Furthermore, stimulation of B cells with anti-CD40 (or anti-IgM, LPS, or CpG) upregulates Btk protein expression (48). Interestingly, Arid3a/Bright is induced in mature resting B cells after BCR and CD40 activation (53), and Arid3a was among the more highly Btk-dependent transcripts downregulated in lupus spleen. The low magnitude of prevention from anti-CD40-triggered B cell proliferation by Syk antagonism, which has been previously observed (37), may provide one explanation why P505-15 is less effective than G-744 in reducing B cell-centered responses. Other mechanisms may include the minimal effect of Syk inhibition preventing Btk activation and the more potent antagonism by P505-15 of Akt compared with G-744.

In addition to pathogenic functions in LN directly mediated by B cells, IC deposition accompanied by macrophage and DC infiltration is a hallmark of inflammatory glomerulonephritis in SLE patients and correlates with poor renal outcome (4). Macrophages play a central role in driving inflammation through recognition of IC via TLRs and Fc $\gamma$ Rs, resulting in effector functions including production of key inflammatory cytokines such as IL1 $\beta$, IL-18, and TNF $\alpha$. In the IFN $\alpha$-accelerated NZB/W_F1 lupus model, glomerulonephritis is characterized by increased proliferative lesions with de novo crescent formation and significantly higher numbers of infiltrating $\mathrm{CD} 11 \mathrm{~b}^{\mathrm{hi}} \mathrm{Gr} 1^{-} \mathrm{CD} 11 \mathrm{c}^{\mathrm{int}} \mathrm{F} 4 / 80^{+}$macrophages than in spontaneously nephritic NZB/W_F1 mice, and these pathogenic findings are suppressed by deletion of macrophages using clodronate (5). We demonstrated previously the crucial role of Btk in controlling IC-mediated arthritis (18). Accordingly, G-744 inhibited Fc $\gamma$ R-triggered TNF $\alpha$ production in mouse macrophages and human monocytes (Table 2), as well as in myeloid cell-dependent CIA in Lewis rat (data not shown). Although not formally shown in the IFN $\alpha$-accelerated model, activating FcRs on circulating hematopoietic cells are necessary for IC-controlled renal pathogenesis in NZB/W_F1 (6). Moreover, activation of $\mathrm{Fc} \gamma \mathrm{R}^{+} \mathrm{CD} 11 \mathrm{~b}^{+}$myeloid cells, including monocytes/macrophages, by glomerular IC deposits is sufficient to initiate glomerulonephritis.

To evaluate the impact of Btk inhibitors on myeloid cell-dependent glomerulonephritis and identify potential Btk-dependent pathway activity in human renal pathogenesis, we characterized the functional consequences of specific Btk inhibition on transcriptional modules that drive renal disease in mice and that also overlapped with characterized renal gene expression patterns of human lupus patients (42). We observed major changes in transcription after treatment with CTX or G-744, likely reflecting amelioration of disease. Surprisingly, Btk inhibition demonstrated a significant overlap in transcriptional response with CTX of $66 \%$ and $48 \%$ in tubulointerstitial and common glomerular/tubulointerstitial gene sets, respectively. Further, G-744 and CTX treatment had overlapping effects in decreasing expression of about $46 \%$ of gene orthologs that were previously identified as being elevated in the kidneys of LN patients. Therapeutic Btk inhibition most significantly abrogated transcripts associated with renal inflammation, cell migration, and myeloid cell infiltration of the glomerulus, but also showed a major effect on tubular gene expression - in particular on molecules associated with membrane trafficking and function that may communicate with the extracellular matrix. These data are consistent with changes in renal gene expression by inhibition of Btk in a nephrotoxic serum transfer model of glomerulonephritis (30). 
Accompanying the gene expression changes was a significant decrease in IgM deposition in glomeruli as well as IgM and IgG staining in proximal convoluted tubules after 4-week inhibitor treatment. In comparison, we did not observe decreased IgG deposition in the glomeruli at this time point, and glomerular $\operatorname{lgG}$ deposition was only moderately increased with disease induction. While we cannot exclude that isotype-specific or select tissue antigen-directed glomerular IgG deposits were missed in our studies due to availability of only formalin-fixed tissue for analyses, the more profound increase in glomerular IgM deposits with disease and concomitant G-744 treatment effects is consistent with its stronger effect on IgM production. Nevertheless, the significant reduction of IgG deposition in proximal convoluted tubules may have contributed to decreased activation of tubular epithelial cells and tubulointerstitial pathology. In this respect, approximately $70 \%$ of LN patients have demonstrable immune aggregates along the renal tubular basement membrane, and the severity of tubulointerstitial changes inversely correlate with renal prognosis (54).

In conclusion, selective inhibition of Btk results in superior efficacy in spontaneous and IFN $\alpha$-accelerated NZB/W_F1 lupus models, compared with BR3-Fc, CTX, and Syk inhibition, by regulating expression of genes that are involved in PC differentiation and $\mathrm{Ab}$ secretion, as well as genes involved in glomerular and tubulointerstitial renal pathology during LN in human patients.

\section{Methods}

Btk enzymatic activity assays and selectivity screens. The in vitro biochemical assay quantified Btk-catalyzed tyrosine phosphorylation of a synthetic peptide, and the kinome selectivity of G-744 was tested in duplicate at $1 \mu \mathrm{M}$ against a panel of 285 active recombinant human kinases. Further details are given in Supplemental Methods.

Drug formulation and dosing. In vitro studies were performed using crystalline compound dissolved in DMSO. Prior to activation, cells were incubated for $30 \mathrm{~min}$ to $1 \mathrm{~h}$ with the concentrations of compound indicated. Compounds used were: G-744 (31) and P505-15 (32). For in vivo studies, G-744 and P505-15 were prepared as described in Supplemental Methods. Both compounds were dosed twice a day (BID) via oral gavage. Murine BR3-Fc or murine anti-ragweed control Abs (generated by Genetech, ref. 55), both containing a mIgG2a Fc, were dosed s.c. or i.p. at $5 \mathrm{mg} / \mathrm{kg}$ or $7.5 \mathrm{mg} / \mathrm{kg} 3$ times per week as indicated. CTX (Baxter) was dosed at $30 \mathrm{mg} / \mathrm{kg}$ i.p. every 10 days.

$B$ cell and monocyte/macrophage in vitro assays. Human B cells or monocytes were isolated from peripheral blood mononuclear cells (PBMCs) by Ficoll-Paque PLUS separation (GE Healthcare) and negatively selected by magnetic cell sorting as described (18). Murine splenic B cells were isolated by negative selection using the mouse B cell isolation kit (Miltenyi). Mouse BM-derived macrophages (BMM) were generated as described (18). Human and murine B cells were stimulated as described in Supplemental Methods. Methods for B cell pBtk, total Btk, pAkt and total Akt determination, CD86 expression, proliferation assays, $\mathrm{Ca}^{2+}$ flux, and survival are described in the Supplemental Methods. TNF $\alpha$ production by Fc $\gamma$ R-activated purified monocytes or mouse BMM cultures was measured by ELISA (Bio-Rad) (18).

Plasmablast generation. Human memory B cells were differentiated into plasmablasts with in vitro stimulation conditions described in Supplemental Methods, followed by flow cytometry analysis at day 7 .

Mice and in vivo BCR activation model. C57BL/6 or Balb/c mice were obtained from Charles River Laboratories; 8-week-old female NZB/W_F1 mice were from the Jackson Laboratory. Mice were housed under pathogen-free conditions until used in a study.

For BCR activation analysis, Balb/c mice were injected i.v. with $150 \mu \mathrm{g} / \mathrm{mouse}$ anti-IgD Abs (catalog $\mathrm{GAM} / \mathrm{IgD}[\mathrm{Fc}] / 7 \mathrm{~S}$, Nordic-MUbios) $1 \mathrm{~h}$ after 1-time drug treatment. CD86 expression on splenic B220 $\mathrm{B}$ cells was quantified $6 \mathrm{~h}$ after anti-IgD stimulation by flow cytometry.

AdIFNa accelerated NZB/W_F1 lupus model. LN was accelerated in 14- to 15-week old NZB/W_F1 mice by injecting i.v. $1.2 \times 10^{8}$ IFN $\alpha$ rAd5-CMV (AdIFN $\alpha$ ) pfu (Elizabeth Davis, Center for Cell \& Gene Therapy, Baylor College of Medicine, Houston, Texas, USA) (55). This treatment typically results in elevated serum IFN $\alpha$ levels of $10 \mathrm{ng} / \mathrm{ml} 3$ weeks after injection and $3 \mathrm{ng} / \mathrm{ml} 6$ weeks after injection compared with LacZ rAd5-CMV control-treated mice. At 3 weeks after AdIFN $\alpha$ injection, mice were treated with vehicle or drugs.

Proteinuria and survival scoring. Proteinuria was determined weekly by colorimetric measurement using dipstick Multistix 10 SG on a Clinitek Status Analyzer (Siemens). Urine protein levels were scored as trace $=0,30 \mathrm{mg} / \mathrm{dl}=1,100 \mathrm{mg} / \mathrm{dl}=2,300 \mathrm{mg} / \mathrm{dl}=3,>300 \mathrm{mg} / \mathrm{dl}=4$, and death $=5$. Progression-free survival was defined as the duration of remission, measured from the first time point when proteinuria was $\leq 300$ $\mathrm{mg} / \mathrm{dl}$, or as survival time if there was no proteinuria progression. 
Histology, histopathologic scoring, IHC, and immunofluorescence. To evaluate renal pathology and splenic GC, kidneys and spleens were formalin fixed and paraffin embedded using routine methods. Renal IC deposition was determined by direct immunofluorescence staining on an autostainer (Dako Universal Autostainer) using Target antigen retrieval as per manufacturer's instructions followed by donkey anti-mouse IgG-AF488 (catalog A-21202) or goat anti-mouse IgM-AF488 (catalog A-21042, Invitrogen). Splenic GCs were detected by IHC on an autostainer (Dako) using Target antigen retrieval, followed by biotinylated anti-lectin-PNA (Vector Labs) and ABC-peroxidase Elite (Dako), and by chromogenic visualization with diaminobenzidine (Dako). All sections were visually evaluated in a blinded fashion by a board-certified pathologist using a Leica DM6000B microscope equipped with a Leica DFC500 camera and LAS software. Isotype or naive control Abs were used to confirm Ab specificity for all immunohistochemical and immunofluorescence stained tissues. Scoring systems for the different stains are described in Supplemental Methods.

Serum Ab ELISAs. Levels of total IgG, IgA, IgM, ANA, and anti-dsDNA Abs were determined by ELISA methodologies as described in detail in Supplemental Methods.

Flow cytometry. Single immune cell suspensions were prepared from spleen or peripheral blood after red blood cell lysis. Cells were counted on a FACS Calibur using Fluoresbrite Calibration Grade 6.0 micron YG microspheres (Polysciences), and flow cytometry staining was carried out as described in Supplemental Methods.

$R N A$ sequencing and differential expression analyses. RNA from frozen spleen and kidney were isolated and analyzed by Illumina HiSeq and bioinformatics analysis performed as described in detail in Supplemental Methods. The raw RNA-sequencing reads and normalized data are available from GEO (accession number GSE72410).

Gene set enrichment analyses and identification of gene sets affected in spleen and kidney of LN mice. Immunology-related gene sets were derived from the immune response in silico (IRIS) microarray compendium and transcript modules from Chiche, et al. $(39,40)$. Significance for GSEA was determined as previously described (56). Gene set statistics were compared with gene set statistics derived from the same dataset with the sample labels permuted 499 times. Gene sets were considered significantly enriched if they showed a more extreme gene set statistic than $>99 \%$ of permutations (i.e. an empirical $P$ value of 0.01 ).

Gene expression changes in spleen were analyzed using datasets published in ref. 41 . To determine changes in gene expression that occur in specific kidney compartments and are regulated in proteinuric mice, we used 2 publicly available datasets: GSE32591 and GSE32583 (42). Bioinformatics methodology is described in Supplemental Methods. Lists of specific genes used for the various expression analyses in spleen and kidney are given in Supplemental Tables 2-6

Statististics. Statistical analysis was performed using PRISM v6.0 (GraphPad). Comparison for each pair was performed using 2-tailed Student's $t$ test with Welch's correction; multiple comparisons with a single control were performed using one-way ANOVA with Dunnett's correction. Survival plots were analyzed by log-rank Mantel-Cox test. $P<0.05$ were considered significant. Statistically significant differences are denoted in figures by glyphs. Absence of these notations indicates no significant differences. The $\mathrm{IC}_{50}$ for each assay was determined with PRISM by plotting the assay output versus the logarithm of the compound concentration using a nonlinear sigmoid with variable slope algorithm with the default curve-fitting parameters in the PRISM software.

Study approval. All animal studies were conducted in compliance with NIH Guide for the Care and Use of Laboratory Animals and were approved by the Genentech Institutional Animal Care and Use Committee.

\section{Author contributions}

AK, YW, and TH designed and performed experiments. JAH analyzed bioinformatics data. ES conducted lupus mice studies. YW and NR prepared RNA and performed RT-PCR analyses. CDA conducted the histology studies. AK and MB performed whole blood assays. JZC, LL, and HW performed PK analyses. KSC, PB, and WBY conceived the G-744 chemical series and synthesis. JJC facilitated G-744 production. JD and WPL supervised in vivo experiments. WPL established the lupus models. JAD and KR conceived the whole blood assays. JAD established the in vivo B cell CD86 assay. JH and JWL managed compound formulation. AJ supervised kinase and selectivity assays. JJC and AJ aided in manuscript assembly. JL performed in vivo B cell CD86 and survival studies. LED, SM, AN, CP, and JW performed Ab ELISA assays. ZL aided in FACS analyses. ML performed Akt phosphorylation assays. ZM generated RNA libraries and RNA sequencing data. MJT and KR conceived and analyzed the bioinformatics studies. KR designed and coordinated the research studies and analyzed the data. KR, MJT, and AK wrote the manuscript. 


\section{Acknowledgments}

The authors thank Christina Chaivorapol for assistance with bioinformatics data analysis; Oleg Mayba for statistical analysis; Pawan Bir Kohli and Steve Sideris for technical help with enzymatic assays; Mercedesz Balazs and Flavius Martin for input on lupus study design; and Margaret Solon, Shari Lau, Andrew Pierce, and Charles Jones III for assistance with histology analysis. Funding for these studies was provided by Genentech.

Address correspondence to: Michael Townsend, Research \& Early Development, Genentech, M/S231c, 1 DNA Way, South San Francisco, California 94080, USA. Phone: 650.225.3239; E-mail: townsem1@ gene.com. Or to: Karin Reif, KARBio, San Francisco, California, USA. Phone: 650.291.7052; E-mail: KARBioSF@gmail.com.

1. Rahman A, Isenberg DA. Systemic lupus erythematosus. N Engl J Med. 2008;358(9):929-939.

2. Anolik JH. B cell biology: implications for treatment of systemic lupus erythematosus. Lupus. 2013;22(4):342-349.

3. Gaipl US, Voll RE, Sheriff A, Franz S, Kalden JR, Herrmann M. Impaired clearance of dying cells in systemic lupus erythematosus. Autoimmun Rev. 2005;4(4):189-194.

4. Nowling TK, Gilkeson GS. Mechanisms of tissue injury in lupus nephritis. Arthritis Res Ther. 2011;13(6):250.

5. Triantafyllopoulou A, et al. Proliferative lesions and metalloproteinase activity in murine lupus nephritis mediated by type I interferons and macrophages. Proc Natl Acad Sci USA. 2010;107(7):3012-3017.

6. Bergtold A, Gavhane A, D’Agati V, Madaio M, Clynes R. FcR-bearing myeloid cells are responsible for triggering murine lupus nephritis. J Immunol. 2006;177(10):7287-7295.

7. Banchereau J, Pascual V. Type I interferon in systemic lupus erythematosus and other autoimmune diseases. Immunity. 2006;25(3):383-392.

8. Rönnblom L. The type I interferon system in the etiopathogenesis of autoimmune diseases. Ups J Med Sci. 2011;116(4):227-237.

9. Looney RJ. B cell-targeted therapies for systemic lupus erythematosus: an update on clinical trial data. Drugs. 2010;70(5):529-540.

10. Rahman AH, Eisenberg RA. The role of toll-like receptors in systemic lupus erythematosus. Springer Semin Immunopathol. 2006;28(2):131-143.

11. Baechler EC, Gregersen PK, Behrens TW. The emerging role of interferon in human systemic lupus erythematosus. Curr Opin Immunol. 2004;16(6):801-807.

12. Santiago-Raber ML, et al. Type-I interferon receptor deficiency reduces lupus-like disease in NZB mice. J Exp Med. 2003;197(6):777-788.

13. Mathian A, Weinberg A, Gallegos M, Banchereau J, Koutouzov S. IFN-alpha induces early lethal lupus in preautoimmune (New Zealand Black x New Zealand White) F1 but not in BALB/c mice. J Immunol. 2005;174(5):2499-2506.

14. Liu Z, Davidson A. IFNa Inducible Models of Murine SLE. Front Immunol. 2013;4:306.

15. Xiong W, Lahita RG. Pragmatic approaches to therapy for systemic lupus erythematosus. Nat Rev Rheumatol. 2014;10(2):97107.

16. Mok CC. Towards new avenues in the management of lupus glomerulonephritis. Nat Rev Rheumatol. 2016;12(4):221-234.

17. Bakshi J, Ismajli M, Rahman A. New therapeutic avenues in SLE. Best Pract Res Clin Rheumatol. 2015;29(6):794-809.

18. Di Paolo JA, et al. Specific Btk inhibition suppresses B cell- and myeloid cell-mediated arthritis. Nat Chem Biol. 2011;7(1):41-50.

19. Brunner C, Müller B, Wirth T. Bruton's Tyrosine Kinase is involved in innate and adaptive immunity. Histol Histopathol. 2005;20(3):945-955.

20. Satterthwaite AB, Witte ON. The role of Bruton's tyrosine kinase in B-cell development and function: a genetic perspective. Immunol Rev. 2000;175:120-127.

21. Tan SL, Liao C, Lucas MC, Stevenson C, DeMartino JA. Targeting the SYK-BTK axis for the treatment of immunological and hematological disorders: recent progress and therapeutic perspectives. Pharmacol Ther. 2013;138(2):294-309.

22. Liu L, Di Paolo J, Barbosa J, Rong H, Reif K, Wong H. Antiarthritis effect of a novel Bruton's tyrosine kinase (BTK) inhibitor in rat collagen-induced arthritis and mechanism-based pharmacokinetic/pharmacodynamic modeling: relationships between inhibition of BTK phosphorylation and efficacy. J Pharmacol Exp Ther. 2011;338(1):154-163.

23. Rankin AL, et al. Selective inhibition of BTK prevents murine lupus and antibody-mediated glomerulonephritis. J Immunol. 2013;191(9):4540-4550.

24. Mina-Osorio P, et al. Suppression of glomerulonephritis in lupus-prone NZB $\times$ NZW mice by RN486, a selective inhibitor of Bruton's tyrosine kinase. Arthritis Rheum. 2013;65(9):2380-2391.

25. Hutcheson J, et al. Modulating proximal cell signaling by targeting Btk ameliorates humoral autoimmunity and end-organ disease in murine lupus. Arthritis Res Ther. 2012;14(6):R243.

26. Honigberg LA, et al. The Bruton tyrosine kinase inhibitor PCI-32765 blocks B-cell activation and is efficacious in models of autoimmune disease and B-cell malignancy. Proc Natl Acad Sci USA. 2010;107(29):13075-13080.

27. Steinberg BJ, Smathers PA, Frederiksen K, Steinberg AD. Ability of the xid gene to prevent autoimmunity in (NZB X NZW)F1 mice during the course of their natural history, after polyclonal stimulation, or following immunization with DNA. JClin Invest. 1982;70(3):587-597.

28. Steinberg EB, Santoro TJ, Chused TM, Smathers PA, Steinberg AD. Studies of congenic MRL-Ipr/Ipr.xid mice. J Immunol. 1983;131(6):2789-2795.

29. Bender AT, et al. Btk inhibition treats TLR7/IFN driven murine lupus. Clin Immunol. 2016;164:65-77.

30. Chalmers SA, et al. Therapeutic Blockade of Immune Complex-Mediated Glomerulonephritis by Highly Selective Inhibition of Bruton's Tyrosine Kinase. Sci Rep. 2016;6:26164. 
31. Barbosa AJM, et al, inventors; Gilead Connecticut, Inc., Genentech, Inc, assignee. Pyridone AZA-Pyridone comppounds methods of use. US Patent 8618107. December 31, 2013.

32. Coffey G, et al. Specific inhibition of spleen tyrosine kinase suppresses leukocyte immune function and inflammation in animal models of rheumatoid arthritis. J Pharmacol Exp Ther. 2012;340(2):350-359.

33. Puri KD, Di Paolo JA, Gold MR. B-cell receptor signaling inhibitors for treatment of autoimmune inflammatory diseases and B-cell malignancies. Int Rev Immunol. 2013;32(4):397-427.

34. So L, Fruman DA. PI3K signalling in B- and T-lymphocytes: new developments and therapeutic advances. Biochem J. 2012;442(3):465-481.

35. Shinners NP, et al. Bruton's tyrosine kinase mediates NF-kappa B activation and B cell survival by B cell-activating factor receptor of the TNF-R family. J Immunol. 2007;179(6):3872-3880.

36. Benson MJ, et al. Modeling the clinical phenotype of BTK inhibition in the mature murine immune system. J Immunol. 2014;193(1):185-197.

37. Hobeika E, et al. CD19 and BAFF-R can signal to promote B-cell survival in the absence of Syk. EMBO J. 2015;34(7):925-939.

38. Dall'era MC, Cardarelli PM, Preston BT, Witte A, Davis JC. Type I interferon correlates with serological and clinical manifestations of SLE. Ann Rheum Dis. 2005;64(12):1692-1697.

39. Abbas AR, et al. Immune response in silico (IRIS): immune-specific genes identified from a compendium of microarray expression data. Genes Immun. 2005;6(4):319-331.

40. Chiche L, et al. Modular transcriptional repertoire analyses of adults with systemic lupus erythematosus reveal distinct type I and type II interferon signatures. Arthritis Rheumatol. 2014;66(6):1583-1595.

41. Shi W, et al. Transcriptional profiling of mouse B cell terminal differentiation defines a signature for antibody-secreting plasma cells. Nat Immunol. 2015;16(6):663-673.

42. Berthier CC, et al. Cross-species transcriptional network analysis defines shared inflammatory responses in murine and human lupus nephritis. J Immunol. 2012;189(2):988-1001.

43. Jourdan M, et al. An in vitro model of differentiation of memory B cells into plasmablasts and plasma cells including detailed phenotypic and molecular characterization. Blood. 2009;114(25):5173-5181.

44. Mathian A, Gallegos M, Pascual V, Banchereau J, Koutouzov S. Interferon- $\alpha$ induces unabated production of short-lived plasma cells in pre-autoimmune lupus-prone (NZB $\times$ NZW)F1 mice but not in BALB/c mice. Eur J Immunol. 2011;41(3):863-872.

45. Nickerson KM, Christensen SR, Cullen JL, Meng W, Luning Prak ET, Shlomchik MJ. TLR9 promotes tolerance by restricting survival of anergic anti-DNA B cells, yet is also required for their activation. J Immunol. 2013;190(4):1447-1456.

46. Jackson SW, et al. Opposing impact of B cell-intrinsic TLR7 and TLR9 signals on autoantibody repertoire and systemic inflammation. J Immunol. 2014;192(10):4525-4532.

47. Shaffer AL, et al. XBP1, downstream of Blimp-1, expands the secretory apparatus and other organelles, and increases protein synthesis in plasma cell differentiation. Immunity. 2004;21(1):81-93

48. Kil LP, et al. Btk levels set the threshold for B-cell activation and negative selection of autoreactive B cells in mice. Blood. 2012;119(16):3744-3756.

49. Fluckiger AC, et al. Btk/Tec kinases regulate sustained increases in intracellular Ca2+ following B-cell receptor activation EMBO J. 1998;17(7):1973-1985

50. Enyedy EJ, Mitchell JP, Nambiar MP, Tsokos GC. Defective FcgammaRIIb1 signaling contributes to enhanced calcium response in B cells from patients with systemic lupus erythematosus. Clin Immunol. 2001;101(2):130-135.

51. Peters AL, Stunz LL, Bishop GA. CD40 and autoimmunity: the dark side of a great activator. Semin Immunol. 2009;21(5):293-300

52. Corneth OB, de Bruijn MJ, Rip J, Asmawidjaja PS, Kil LP, Hendriks RW. Enhanced Expression of Bruton's Tyrosine Kinase in B Cells Drives Systemic Autoimmunity by Disrupting T Cell Homeostasis. J Immunol. 2016;197(1):58-67.

53. Ratliff ML, Ward JM, Merrill JT, James JA, Webb CF. Differential expression of the transcription factor ARID3a in lupus patient hematopoietic progenitor cells. J Immunol. 2015;194(3):940-949.

54. Yung S, Chan TM. Autoantibodies and resident renal cells in the pathogenesis of lupus nephritis: getting to know the unknown. Clin Dev Immunol. 2012;2012:139365.

55. Lin W, et al. Dual B cell immunotherapy is superior to individual anti-CD20 depletion or BAFF blockade in murine models of spontaneous or accelerated lupus. Arthritis Rheumatol. 2015;67(1):215-224.

56. Jiang Z, Gentleman R. Extensions to gene set enrichment. Bioinformatics. 2007;23(3):306-313. 